\title{
Demethylative Lactonization Provides a Shortcut to High-Yielding Syntheses of Lamellarins
}

\author{
Robin Klintworth, Charles B. de Koning and Joseph P. Michael* \\ Molecular Sciences Institute, School of Chemistry, University of the Witwatersrand, Johannesburg, PO Wits \\ 2050, South Africa \\ *Email: joseph.michael@wits.ac.za
}

\section{Contents}

1. Tabulated Comparisons with Reported ${ }^{1} \mathrm{H}$ and ${ }^{13} \mathrm{C}$ NMR Spectroscopic Data for Various Lamellarins ... S2

2. References S6

3. NMR Spectra S7 


\section{Tabulated Comparisons with Reported ${ }^{1} \mathrm{H}$ and ${ }^{13} \mathrm{C}$ NMR Spectroscopic Data for Various Lamellarins}

Table S1. Comparison of ${ }^{1} \mathrm{H}$ and ${ }^{13} \mathrm{C}$ NMR spectroscopic data for lamellarin $\mathrm{G}$ trimethyl ether (6) with reported values

\begin{tabular}{|c|c|c|c|c|c|}
\hline \multicolumn{3}{|c|}{${ }^{1} \mathrm{H}$ NMR DATA } & \multicolumn{3}{|c|}{${ }^{13}$ C NMR DATA } \\
\hline This work & Kumar et al. ${ }^{1}$ & Colligs et al. ${ }^{2}$ & This work & Kumar et al. ${ }^{1}$ & Colligs et al. ${ }^{2}$ \\
\hline $500 \mathrm{MHz}$ & $400 \mathrm{MHz}$ & $400 \mathrm{MHz}$ & $126 \mathrm{MHz}$ & $100 \mathrm{MHz}$ & $100 \mathrm{MHz}$ \\
\hline $\mathrm{CDCl}_{3}$ & $\mathrm{CDCl}_{3}$ & $\mathrm{CDCl}_{3}$ & $\mathrm{CDCl}_{3}$ & $\mathrm{CDCl}_{3}$ & $\mathrm{CDCl}_{3}$ \\
\hline $\begin{array}{l}7.13(\mathrm{dd}, J=8.5,2.0 \\
\mathrm{Hz}, 1 \mathrm{H})\end{array}$ & $\begin{array}{l}7.11(\mathrm{~d}, J=8.2 \mathrm{~Hz}, \\
1 \mathrm{H})\end{array}$ & $\begin{array}{l}7.11(\mathrm{dd}, J=8.1,1.8 \\
\mathrm{Hz}, 1 \mathrm{H})\end{array}$ & 155.5 & 155.6 & 155.5 \\
\hline $\begin{array}{l}7.08(\mathrm{~d}, J=8.0 \mathrm{~Hz} \\
1 \mathrm{H})\end{array}$ & $7.07(\mathrm{~d}, J=8 \mathrm{~Hz}, 1 \mathrm{H})$ & $\begin{array}{l}7.07(\mathrm{~d}, J=8.1 \mathrm{~Hz}, \\
1 \mathrm{H})\end{array}$ & 149.8 & 149.7 & 149.7 \\
\hline $\begin{array}{l}7.06(\mathrm{~d}, J=1.5 \mathrm{~Hz}, \\
1 \mathrm{H})\end{array}$ & $7.04(\mathrm{~d}, J=2 \mathrm{~Hz}, 1 \mathrm{H})$ & $\begin{array}{l}7.05(\mathrm{~d}, J=1.8 \mathrm{~Hz}, \\
1 \mathrm{H})\end{array}$ & 149.0 & 149.0 & 149.0 \\
\hline $6.90(\mathrm{~s}, 1 \mathrm{H})$ & $6.90(\mathrm{~s}, 1 \mathrm{H})$ & $6.90(\mathrm{~s}, 1 \mathrm{H})$ & 148.9 & 148.84 & 148.8 \\
\hline $6.76(\mathrm{~s}, 1 \mathrm{H})$ & $6.76(\mathrm{~s}, 1 \mathrm{H})$ & $6.78(\mathrm{~s}, 1 \mathrm{H})$ & 148.8 & 148.81 & 148.8 \\
\hline $6.72(\mathrm{~s}, 1 \mathrm{H})$ & $6.71(\mathrm{~s}, 1 \mathrm{H})$ & $6.71(\mathrm{~s}, 1 \mathrm{H})$ & 147.5 & 147.5 & 147.5 \\
\hline $6.67(s, 1 H)$ & $6.66(\mathrm{~s}, 1 \mathrm{H})$ & $6.66(\mathrm{~s}, 1 \mathrm{H})$ & 146.1 & 146.1 & 146.1 \\
\hline $\begin{array}{l}4.79(2 \times \text { quintet, } J= \\
6.6 \mathrm{~Hz}, 2 \mathrm{H})\end{array}$ & 4.86-4.7 (m, 2H) & $4.85-4.73(\mathrm{~m}, 2 \mathrm{H})$ & 145.5 & 145.5 & 145.5 \\
\hline $3.96(\mathrm{~s}, 3 \mathrm{H})$ & $3.95(\mathrm{~s}, 3 \mathrm{H})$ & $3.95(\mathrm{~s}, 3 \mathrm{H})$ & 135.9 & 136.0 & 135.9 \\
\hline $3.90(\mathrm{~s}, 3 \mathrm{H})$ & $3.89(\mathrm{~s}, 3 \mathrm{H})$ & $3.89(\mathrm{~s}, 3 \mathrm{H})$ & 128.2 & 128.2 & 128.2 \\
\hline $3.88(\mathrm{~s}, 3 \mathrm{H})$ & $3.88(\mathrm{~s}, 3 \mathrm{H})$ & $3.87(\mathrm{~s}, 3 \mathrm{H})$ & 128.0 & 128.0 & 128.0 \\
\hline $3.87(\mathrm{~s}, 3 \mathrm{H})$ & $3.86(\mathrm{~s}, 3 \mathrm{H})$ & $3.86(\mathrm{~s}, 3 \mathrm{H})$ & 126.6 & 126.6 & 126.6 \\
\hline $3.46(\mathrm{~s}, 3 \mathrm{H})$ & $3.45(\mathrm{~s}, 3 \mathrm{H})$ & $3.45(\mathrm{~s}, 3 \mathrm{H})$ & 123.6 & 123.6 & 123.6 \\
\hline $3.37(\mathrm{~s}, 3 \mathrm{H})$ & $3.36(\mathrm{~s}, 3 \mathrm{H})$ & $3.36(\mathrm{~s}, 3 \mathrm{H})$ & 120.1 & 120.0 & 120.0 \\
\hline $\begin{array}{l}3.12(\mathrm{t}, J=6.6 \mathrm{~Hz}, \\
2 \mathrm{H})\end{array}$ & $\begin{array}{l}3.12(\mathrm{t}, J=7.2 \mathrm{~Hz}, \\
2 \mathrm{H})\end{array}$ & $3.12(m, 2 H)$ & 114.8 & 114.8 & 114.7 \\
\hline & & & 114.0 & 114.0 & 114.0 \\
\hline & & & 113.8 & 113.8 & 113.7 \\
\hline & & & 111.9 & 111.8 & 111.9 \\
\hline & & & 111.0 & 111.0 & 111.0 \\
\hline & & & 110.3 & 110.3 & 110.3 \\
\hline & & & 108.7 & 108.6 & 108.7 \\
\hline & & & 104.5 & 104.5 & 104.5 \\
\hline & & & 100.5 & 100.5 & 100.5 \\
\hline & & & 56.3 & 56.24 & 56.2 \\
\hline & & & 56.2 & 56.16 & 56.1 \\
\hline & OMe & & 56.1 & 56.1 & 56.0 \\
\hline & 6 & & 55.9 & 55.9 & 55.9 \\
\hline & & & 55.5 & 55.5 & 55.4 \\
\hline & & & 55.2 & 55.2 & 55.2 \\
\hline & & & 42.4 & 42.4 & 42.4 \\
\hline & & & 28.7 & 28.7 & 28.7 \\
\hline
\end{tabular}


Table S2. Comparison of ${ }^{1} \mathrm{H}$ and ${ }^{13} \mathrm{C}$ NMR spectroscopic data for lamellarin A4 (3) with reported values

\begin{tabular}{|c|c|c|c|c|c|}
\hline \multicolumn{3}{|c|}{${ }^{1} \mathrm{H}$ NMR DATA } & \multicolumn{3}{|c|}{${ }^{13}$ C NMR DATA } \\
\hline This work & Kumar et al. ${ }^{1}$ & Plisson et al. ${ }^{3}$ & This work & $\begin{array}{l}\text { Kumar et } \\
\text { al. }^{2}\end{array}$ & $\begin{array}{l}\text { Plisson et } \\
\text { al. }^{3}\end{array}$ \\
\hline $400 \mathrm{MHz}$ & $400 \mathrm{MHz}$ & $600 \mathrm{MHz}$ & $101 \mathrm{MHz}$ & $100 \mathrm{MHz}$ & $125 \mathrm{MHz}$ \\
\hline DMSO- $d_{6}$ & DMSO- $d_{6}$ & DMSO- $d_{6}$ & DMSO- $d_{6}$ & DMSO- $d_{6}$ & DMSO- $d_{6}$ \\
\hline $9.58(\mathrm{~s}, 1 \mathrm{H})$ & $9.55(\mathrm{~s}, 1 \mathrm{H})$ & $9.58(\mathrm{~s}, 1 \mathrm{H})$ & 154.9 & 154.9 & 154.4 \\
\hline $9.37(\mathrm{~s}, 1 \mathrm{H})$ & $9.37(\mathrm{~s}, 1 \mathrm{H})$ & $9.37(\mathrm{~s}, 1 \mathrm{H})$ & 146.5 & 146.5 & 146.0 \\
\hline \multirow{2}{*}{$9.11(\mathrm{~s}, 2 \mathrm{H})$} & \multirow{2}{*}{$9.10(\mathrm{~s}, 2 \mathrm{H})$} & $9.10(\mathrm{~s}, 1 \mathrm{H})$ & 146.4 & 146.4 & 145.9 \\
\hline & & $9.08(\mathrm{~s}, 1 \mathrm{H})$ & 146.3 & 146.3 & 145.8 \\
\hline $8.81(\mathrm{~s}, 1 \mathrm{H})$ & $8.82(\mathrm{~s}, 1 \mathrm{H})$ & $8.81(\mathrm{~s}, 1 \mathrm{H})$ & 145.6 & 145.6 & 145.1 \\
\hline $8.61(\mathrm{~s}, 1 \mathrm{H})$ & $8.63(\mathrm{~s}, 1 \mathrm{H})$, & $8.61(\mathrm{~s}, 1 \mathrm{H})$ & 145.1 & 145.1 & 144.6 \\
\hline $\begin{array}{l}6.91(\mathrm{~d}, J=8.0 \mathrm{~Hz}, \\
1 \mathrm{H})\end{array}$ & $6.87(\mathrm{~d}, J=8 \mathrm{~Hz}, 2 \mathrm{H})$ & $6.90(\mathrm{~d}, 1 \mathrm{H})$ & 144.0 & 144.0 & 143.5 \\
\hline $6.74(\mathrm{~s}, 1 \mathrm{H})$ & $6.72(s, 1 H)$ & $6.73(\mathrm{~s}, 1 \mathrm{H})$ & 142.4 & 142.2 & 141.9 \\
\hline $\begin{array}{l}6.70(\mathrm{~d}, J=2.0 \mathrm{~Hz}, \\
1 \mathrm{H})\end{array}$ & $6.67(\mathrm{~d}, J=2 \mathrm{~Hz}, 1 \mathrm{H})$ & $6.69(\mathrm{~d}, 1 \mathrm{H})$ & 136.4 & 136.4 & 135.9 \\
\hline $6.69(\mathrm{~s}, 1 \mathrm{H})$ & $6.66(\mathrm{~s}, 1 \mathrm{H})$ & $6.68(\mathrm{~s}, 1 \mathrm{H})$ & 127.9 & 127.9 & 127.5 \\
\hline $\begin{array}{l}6.63(\mathrm{dd}, J=8.0,2.0 \\
\mathrm{Hz}, 1 \mathrm{H})\end{array}$ & $\begin{array}{l}6.59(\mathrm{dd}, J=8,1.6 \\
\mathrm{Hz}, 1 \mathrm{H})\end{array}$ & $6.61(\mathrm{~d}, 1 \mathrm{H})$ & 126.2 & 126.2 & 125.7 \\
\hline $6.53(\mathrm{~s}, 1 \mathrm{H})$ & $6.50(\mathrm{~s}, 1 \mathrm{H})$ & $6.53(\mathrm{~s}, 1 \mathrm{H})$ & 125.8 & 125.8 & 125.4 \\
\hline $6.47(\mathrm{~s}, 1 \mathrm{H})$ & $6.44(\mathrm{~s}, 1 \mathrm{H})$ & $6.46(\mathrm{~s}, 1 \mathrm{H})$ & 121.7 & 121.17 & 121.2 \\
\hline $\begin{array}{l}4.60 \text { and } 4.54(2 \times \\
\text { quintets, } J \text { ca } 6.8 \mathrm{~Hz} \text {, } \\
2 \mathrm{H})\end{array}$ & $4.63-4.47(\mathrm{~m}, 2 \mathrm{H})$ & $4.60,4.52(2 \times m, 2 H)$ & 119.0 & 118.9 & 118.5 \\
\hline $\begin{array}{l}2.95(\mathrm{brt}, J=6.8 \mathrm{~Hz}, \\
2 \mathrm{H})\end{array}$ & $\begin{array}{l}2.92(\mathrm{t}, J=6.4 \mathrm{~Hz}, \\
2 \mathrm{H})\end{array}$ & $2.93(t ?, 2 \mathrm{H})$ & 117.9 & 117.9 & 117.4 \\
\hline & & & 117.2 & 117.2 & 116.7 \\
\hline & & & 115.5 & 115.5 & 115.0 \\
\hline & & & 115.1 & 115.1 & 114.6 \\
\hline & & & 113.9 & 113.8 & 113.4 \\
\hline & & & 112.6 & 112.6 & 112.1 \\
\hline & & & 109.7 & 109.7 & 109.2 \\
\hline & & & 109.3 & 109.3 & 108.8 \\
\hline & U & & 103.7 & 103.6 & 103.2 \\
\hline & 3 & & 42.7 & 42.4 & 42.0 \\
\hline & & & 28.3 & 28.3 & 27.8 \\
\hline
\end{tabular}


Table S3. Comparison of ${ }^{1} \mathrm{H}$ and ${ }^{13} \mathrm{C}$ NMR spectroscopic data for lamellarin $\mathrm{D}$ trimethyl ether (7) with reported values

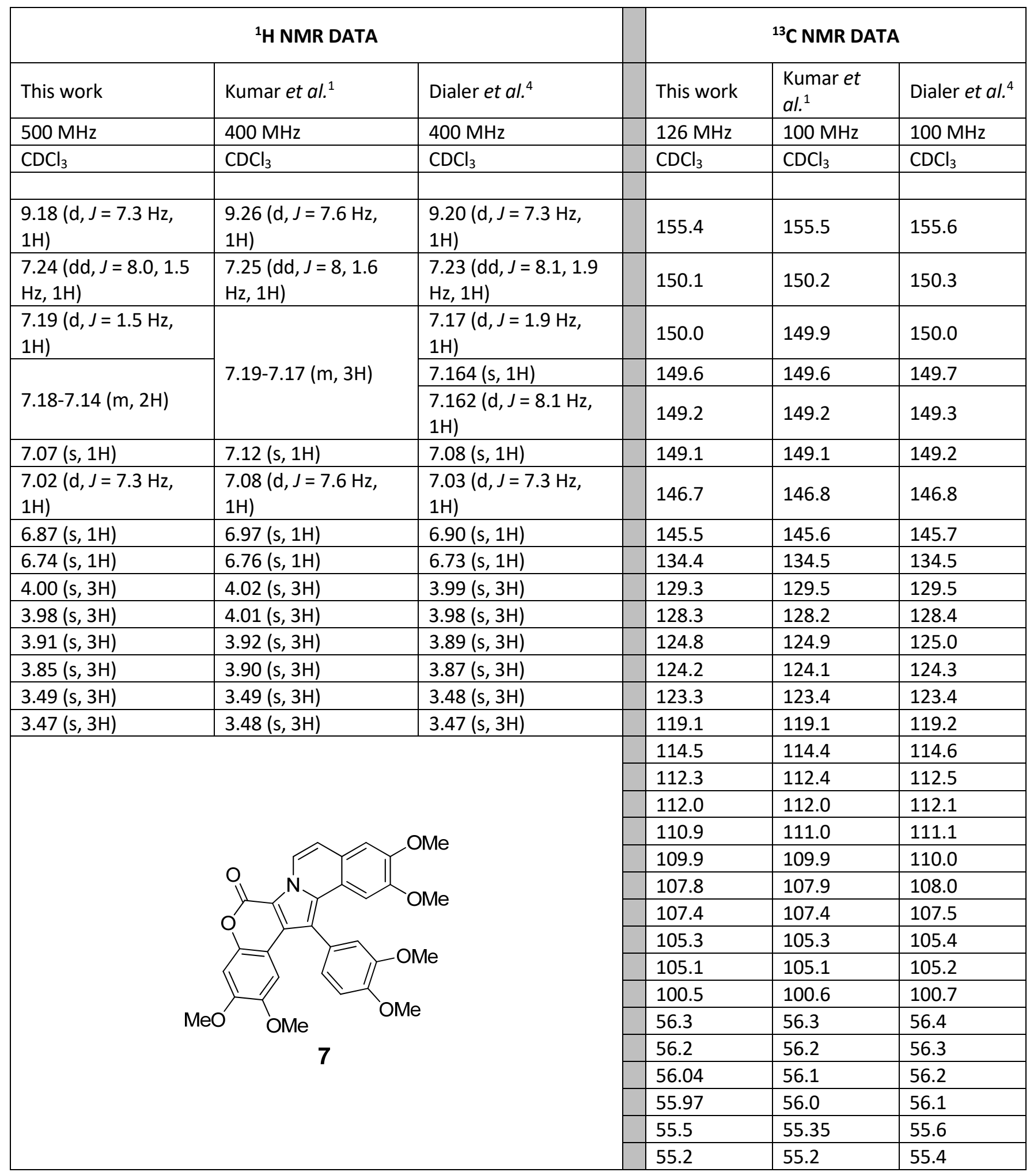


Table S4. Comparison of ${ }^{1} \mathrm{H}$ and ${ }^{13} \mathrm{C}$ NMR spectroscopic data for lamellarin $\mathrm{H}$ (5) with reported values

\begin{tabular}{|c|c|c|c|c|c|}
\hline \multicolumn{3}{|c|}{${ }^{1} \mathrm{H}$ NMR DATA } & \multicolumn{3}{|c|}{${ }^{13}$ C NMR DATA } \\
\hline This work & Kumar et al. ${ }^{1}$ & Dialer et al. ${ }^{4}$ & This work & $\begin{array}{l}\text { Kumar et } \\
\text { al. }{ }^{1}\end{array}$ & Dialer et al. ${ }^{4}$ \\
\hline $400 \mathrm{MHz}$ & $400 \mathrm{MHz}$ & $400 \mathrm{MHz}$ & $101 \mathrm{MHz}$ & $100 \mathrm{MHz}$ & $100 \mathrm{MHz}$ \\
\hline DMSO- $d_{6}$ & DMSO- $d_{6}$ & DMSO- $d_{6}$ & DMSO- $d_{6}$ & DMSO- $d_{6}$ & DMSO- $d_{6}$ \\
\hline $10.04(\mathrm{~s}, 1 \mathrm{H})$ & $10.00(\mathrm{~s}, 1 \mathrm{H})$ & 9.99 (br s, 1H) & 154.9 & 155.0 & 154.5 \\
\hline $9.81(\mathrm{~s}, 1 \mathrm{H})$ & $9.77(\mathrm{~s}, 1 \mathrm{H})$ & $9.76(\mathrm{br} \mathrm{s}, 1 \mathrm{H})$ & 148.1 & 148.1 & 147.7 \\
\hline $9.47(\mathrm{~s}, 1 \mathrm{H})$ & $9.43(\mathrm{~s}, 1 \mathrm{H})$ & $9.42(\mathrm{br} \mathrm{s}, 1 \mathrm{H})$ & 147.3 & 147.3 & 146.8 \\
\hline $9.26(\mathrm{~s}, 1 \mathrm{H})$ & $9.22(\mathrm{~s}, 1 \mathrm{H})$ & \multirow{2}{*}{9.20 (br s, 2H) } & 147.0 & 147.0 & 146.6 \\
\hline $9.25(\mathrm{~s}, 1 \mathrm{H})$ & $9.21(\mathrm{~s}, 1 \mathrm{H})$ & & 146.7 & 146.7 & 146.2 \\
\hline $\begin{array}{l}9.05(\mathrm{~d}, J=7.3 \mathrm{~Hz}, \\
1 \mathrm{H})\end{array}$ & $\begin{array}{l}8.99(\mathrm{~d}, J=7.6 \mathrm{~Hz}, \\
1 \mathrm{H})\end{array}$ & $\begin{array}{l}8.99(\mathrm{~d}, J=7.3 \mathrm{~Hz}, \\
1 \mathrm{H})\end{array}$ & 146.0 & 146.0 & 145.5 \\
\hline $8.96(\mathrm{~s}, 1 \mathrm{H})$ & $8.92(\mathrm{~s}, 1 \mathrm{H})$ & $8.91(\mathrm{br} \mathrm{s}, 1 \mathrm{H})$ & 145.7 & 145.7 & 145.3 \\
\hline $\begin{array}{l}7.21(\mathrm{~d}, J=6.0 \mathrm{~Hz}, \\
1 \mathrm{H})\end{array}$ & $7.15(\mathrm{~d}, J=8 \mathrm{~Hz}, 1 \mathrm{H})$ & $\begin{array}{l}7.14(\mathrm{~d}, J=7.3 \mathrm{~Hz}, \\
1 \mathrm{H})\end{array}$ & 142.5 & 142.6 & 142.1 \\
\hline $7.19(\mathrm{~s}, 1 \mathrm{H})$ & $7.14(\mathrm{~s}, 1 \mathrm{H})$ & $7.13(\mathrm{~s}, 1 \mathrm{H})$ & 134.4 & 134.4 & 133.9 \\
\hline $\begin{array}{l}7.06(\mathrm{~d}, J=7.6 \mathrm{~Hz}, \\
1 \mathrm{H})\end{array}$ & $6.99(\mathrm{~d}, J=8 \mathrm{~Hz}, 1 \mathrm{H})$ & $\begin{array}{l}6.99(\mathrm{~d}, J=8.0 \mathrm{~Hz}, \\
1 \mathrm{H})\end{array}$ & 129.3 & 129.3 & 128.9 \\
\hline $7.02(\mathrm{~s}, 1 \mathrm{H})$ & $6.96(\mathrm{~s}, 1 \mathrm{H})$ & $6.95(\mathrm{~s}, 1 \mathrm{H})$ & 125.9 & 125.9 & 125.4 \\
\hline $6.87(s, 1 \mathrm{H})$ & $6.83(\mathrm{~s}, 1 \mathrm{H})$ & $6.81(\mathrm{~s}, 1 \mathrm{H})$ & 124.2 & 124.2 & 123.8 \\
\hline $\begin{array}{l}6.86(\mathrm{~d}, J=2.0 \mathrm{~Hz}, \\
1 \mathrm{H})\end{array}$ & $6.79(\mathrm{~d}, J=2 \mathrm{~Hz}, 1 \mathrm{H})$ & $\begin{array}{l}6.79(\mathrm{~d}, J=2.0 \mathrm{~Hz}, \\
1 \mathrm{H})\end{array}$ & 121.9 & 121.9 & 121.4 \\
\hline $\begin{array}{l}6.78(\mathrm{dd}, J=7.9,2.0 \\
\mathrm{Hz}, 1 \mathrm{H})\end{array}$ & $\begin{array}{l}6.71(\mathrm{dd}, J=8.0,2.0 \\
\mathrm{Hz}, 1 \mathrm{H})\end{array}$ & $\begin{array}{l}6.71(\mathrm{dd}, J=8.0,2.0 \\
\mathrm{Hz}, 1 \mathrm{H})\end{array}$ & 121.6 & 121.6 & 121.2 \\
\hline $6.64(\mathrm{~s}, 1 \mathrm{H})$ & $6.57(\mathrm{~s}, 1 \mathrm{H})$ & $6.57(\mathrm{~s}, 1 \mathrm{H})$ & 118.6 & 118.6 & 118.1 \\
\hline \multirow{10}{*}{\multicolumn{3}{|c|}{ (c) }} & 118.0 & 118.0 & 117.6 \\
\hline & & & 117.5 & 117.5 & 117.0 \\
\hline & & & 113.0 & 113.0 & 112.6 \\
\hline & & & 111.87 & 111.9 & 111.41 \\
\hline & & & 111.85 & 111.9 & 111.39 \\
\hline & & & 110.1 & 110.1 & 109.62 \\
\hline & & & 110.0 & 110.0 & 109.56 \\
\hline & & & 109.3 & 109.2 & 108.8 \\
\hline & & & 106.8 & 106.8 & 106.3 \\
\hline & & & 103.8 & 103.8 & 103.3 \\
\hline
\end{tabular}




\section{References}

1. Kumar, V.; Awasthi, A.; Salam, A.; Khan, T. Scalable Total Syntheses of Some Natural and Unnatural Lamellarins: Application of a One-Pot Domino Process for Regioselective Access to the Central 1,2,4-Trisubstituted Pyrrole Core. J. Org. Chem. 2019, 84, 11596-11603.

2. Colligs, V. C.; Dialer, C.; Opatz, T. Synthesis of Lamellarin G Trimethyl Ether by von Miller-PlöchlType Cyclocondensation. Eur. J. Org. Chem. 2018, 4064-4070.

3. Plisson, F.; Huang, X.-C.; Zhang, H.; Khalil, Z.; Capon, R. J. Lamellarins as Inhibitors of PGlycoprotein-Mediated Multidrug Resistance in a Human Colon Cancer Cell Line. Chem. - Asian J. 2012, 7, 1616-1623.

4. Dialer, C.; Imbri, D.; Hansen, S. P.; Opatz, T. Synthesis of Lamellarin D Trimethyl Ether and Lamellarin H via 6r-Electrocyclization. J. Org. Chem. 2015, 80, 11605-11610. 


\section{NMR Spectra}

${ }^{1} \mathrm{H}$ NMR spectrum of 6,7-dimethoxy-3,4-dihydroisoquinoline-1(2H)-thione (12) $\left(300 \mathrm{MHz}, \mathrm{CDCl}_{3}\right)$

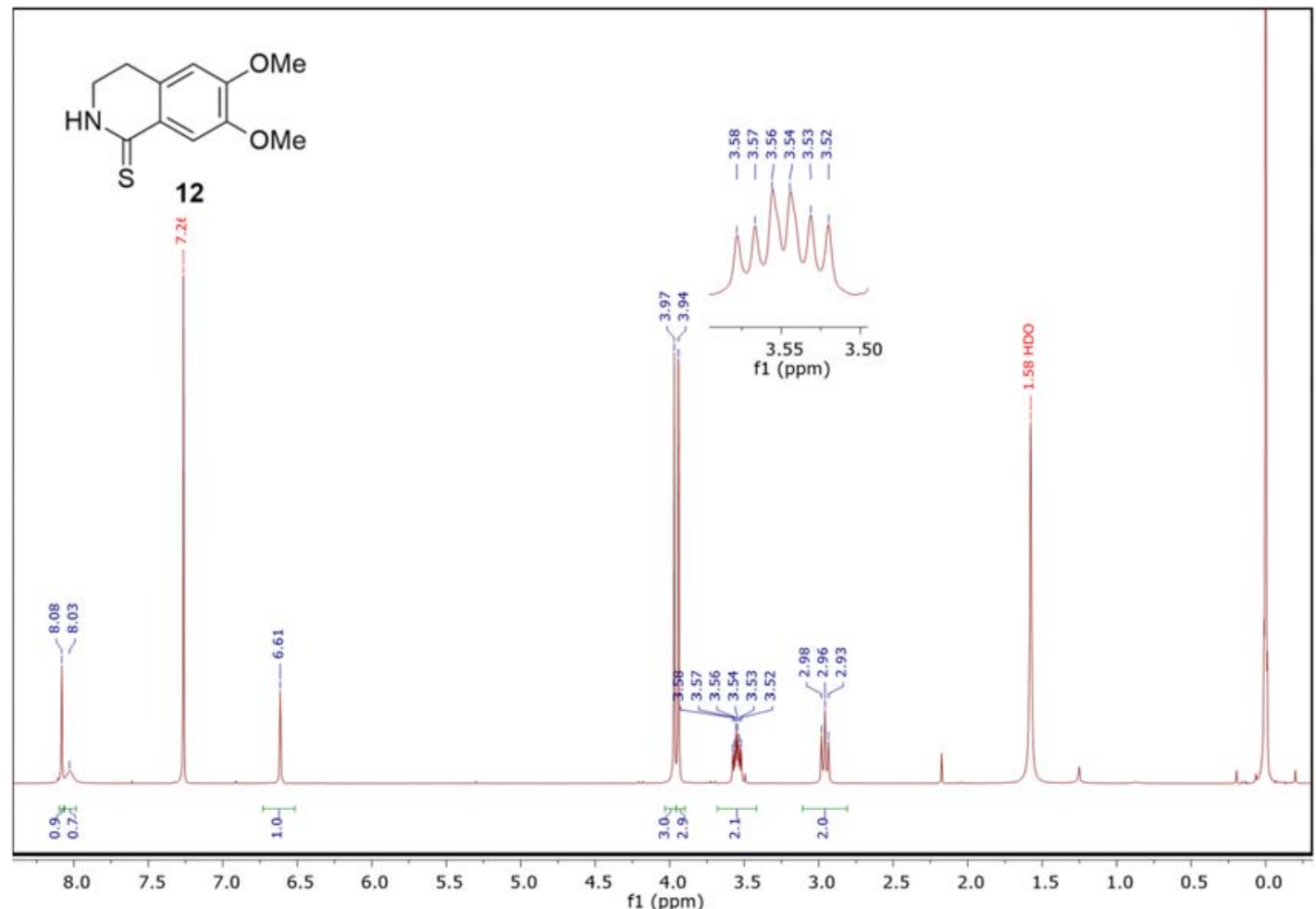

${ }^{13} \mathrm{C}\left\{{ }^{1} \mathrm{H}\right\}$ NMR spectrum of 6,7-dimethoxy-3,4-dihydroisoquinoline-1(2H)-thione (12) (126 MHz, DMSO- $\left.d_{6}\right)$

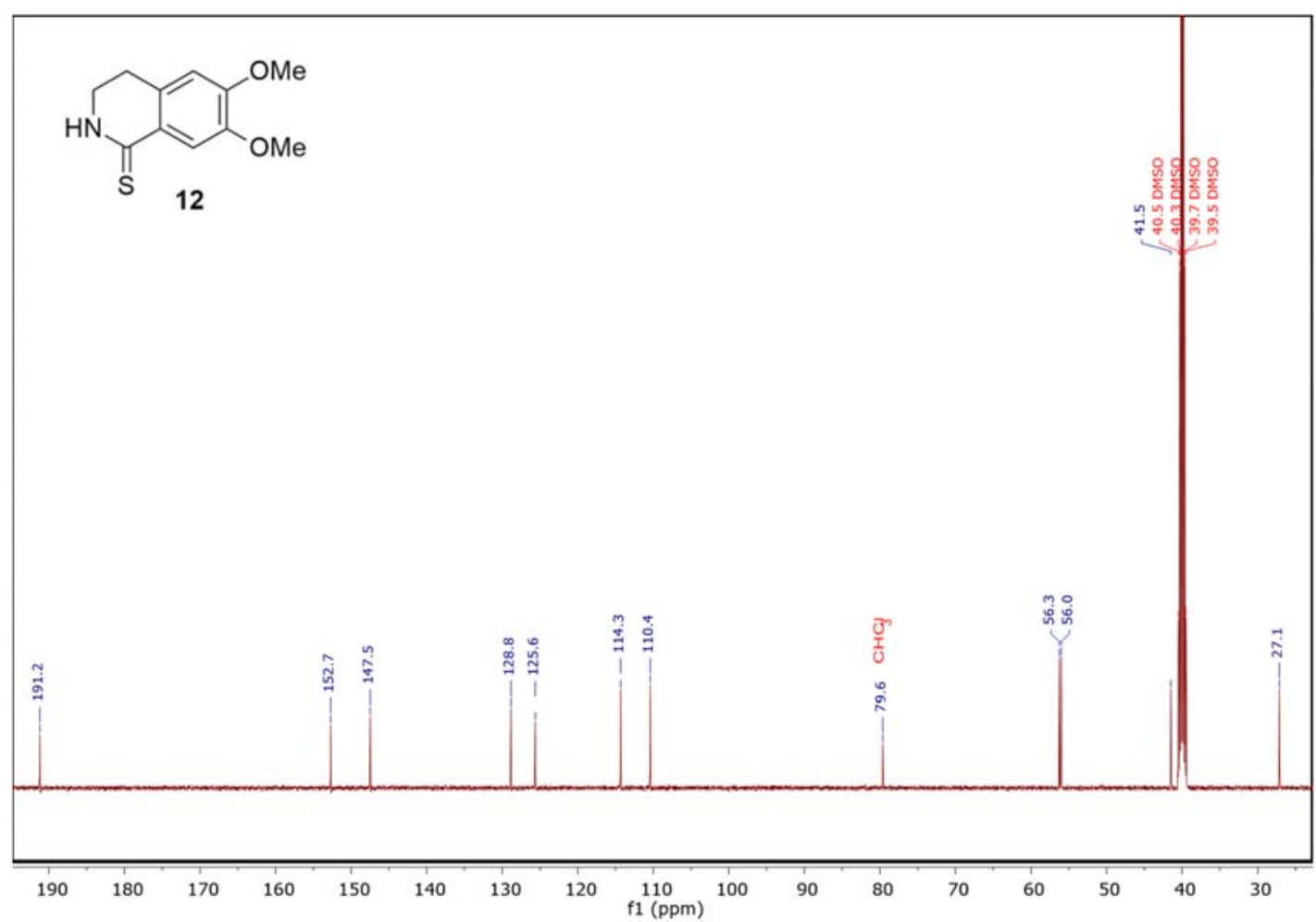


${ }^{1} \mathrm{H}$ NMR spectrum of 2-(3,4-dimethoxyphenyl)-1-(2,4,5-trimethoxyphenyl)ethanone (18) $\left(300 \mathrm{MHz}^{\mathrm{CDCl}} \mathrm{CD}_{3}\right.$

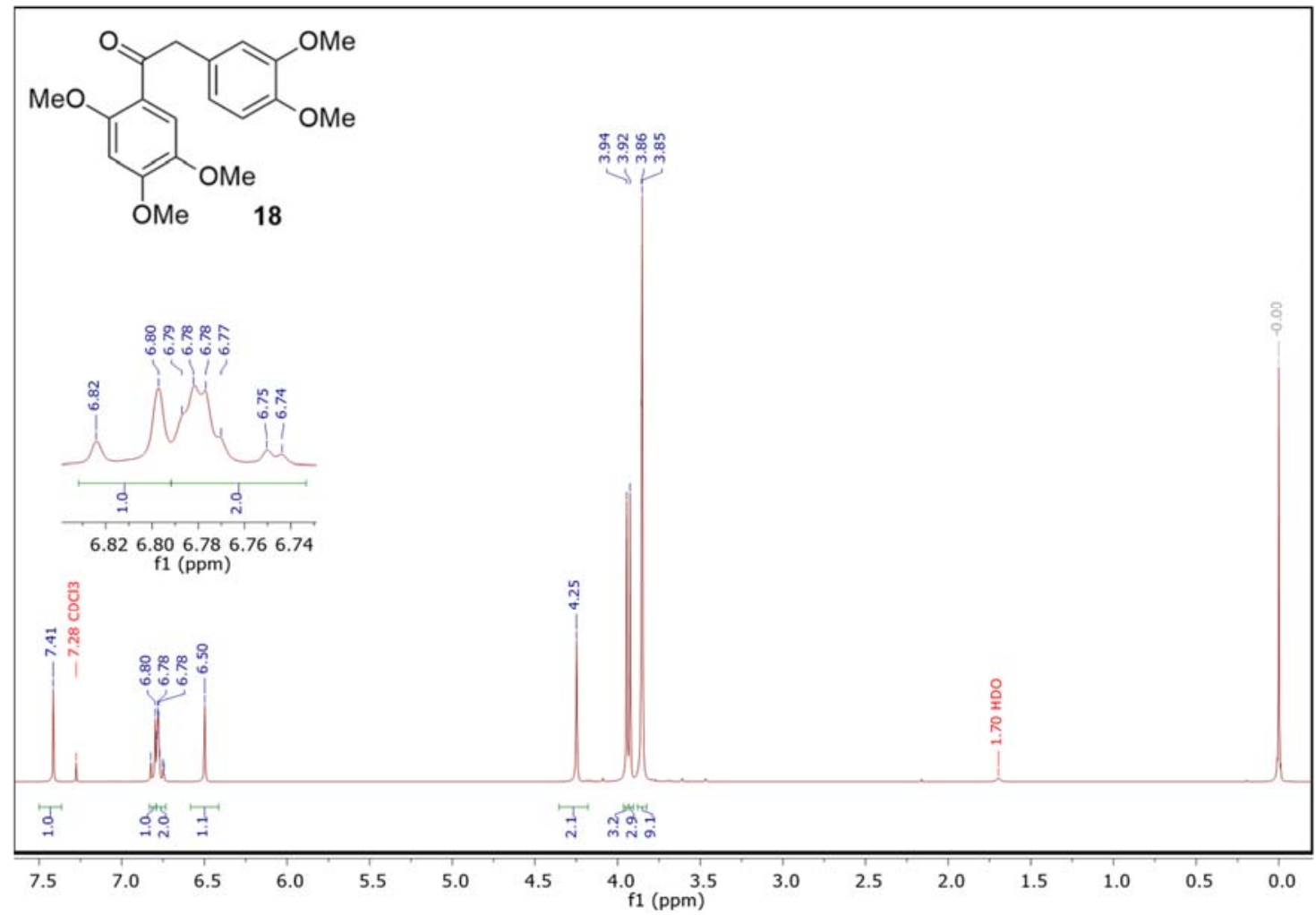

${ }^{13} \mathrm{C}\left\{{ }^{1} \mathrm{H}\right\}$ NMR spectrum of 2-(3,4-dimethoxyphenyl)-1-(2,4,5-trimethoxyphenyl)ethanone (18) (75 $\left.\mathrm{MHz}, \mathrm{CDCl}_{3}\right)$

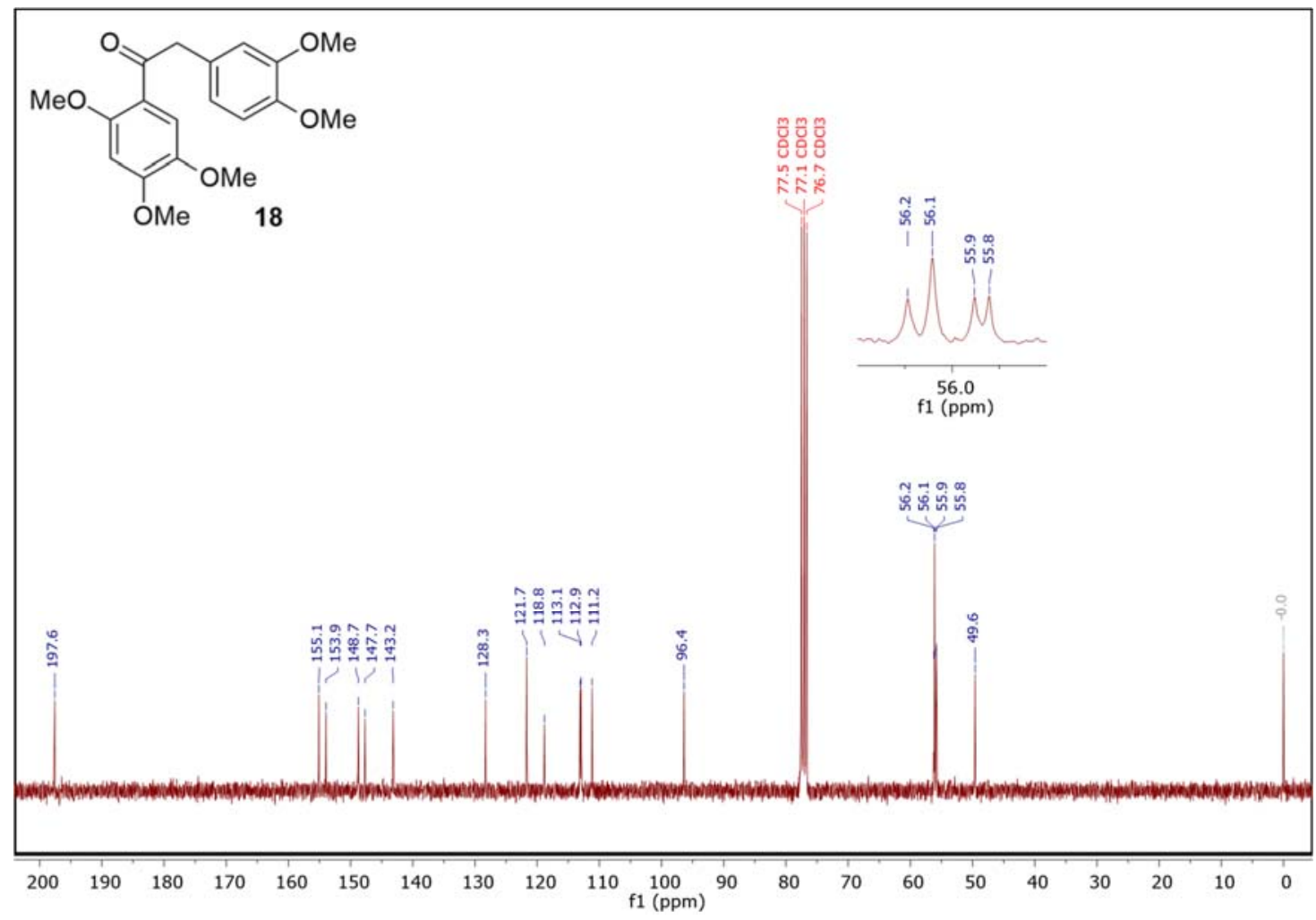


${ }^{1} \mathrm{H}$ NMR spectrum of 2-bromo-2-(3,4-dimethoxyphenyl)-1-(2,4,5-trimethoxyphenyl)ethanone (19) $\left(500 \mathrm{MHz}^{\left.-\mathrm{CDCl}_{3}\right)}\right.$

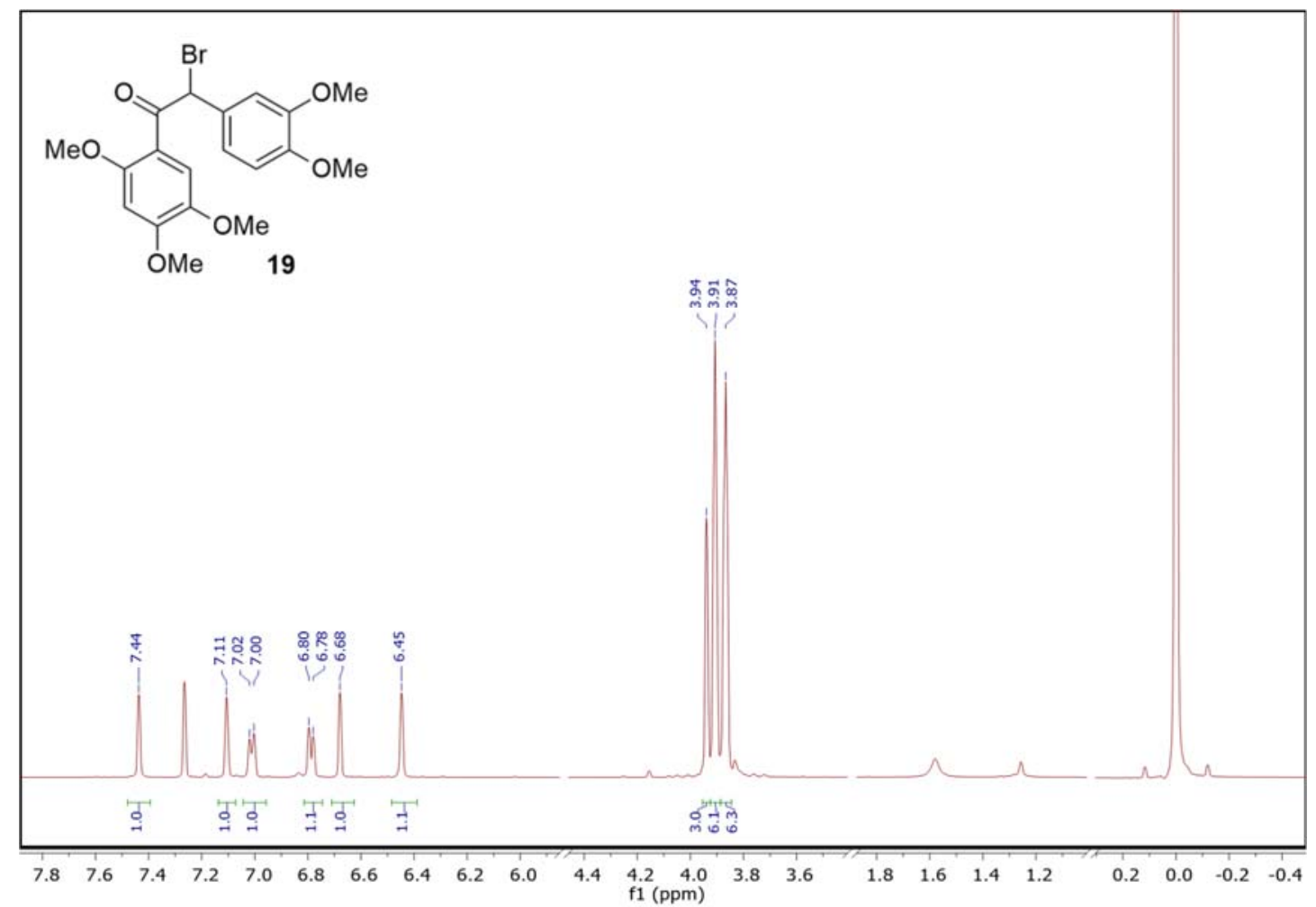

${ }^{13} \mathrm{C}\left\{{ }^{1} \mathrm{H}\right\}$ NMR spectrum of 2-bromo-2-(3,4-dimethoxyphenyl)-1-(2,4,5-trimethoxyphenyl)ethanone (19) (126 $\left.\mathrm{MHz} \mathrm{CDCl}{ }_{3}\right)$

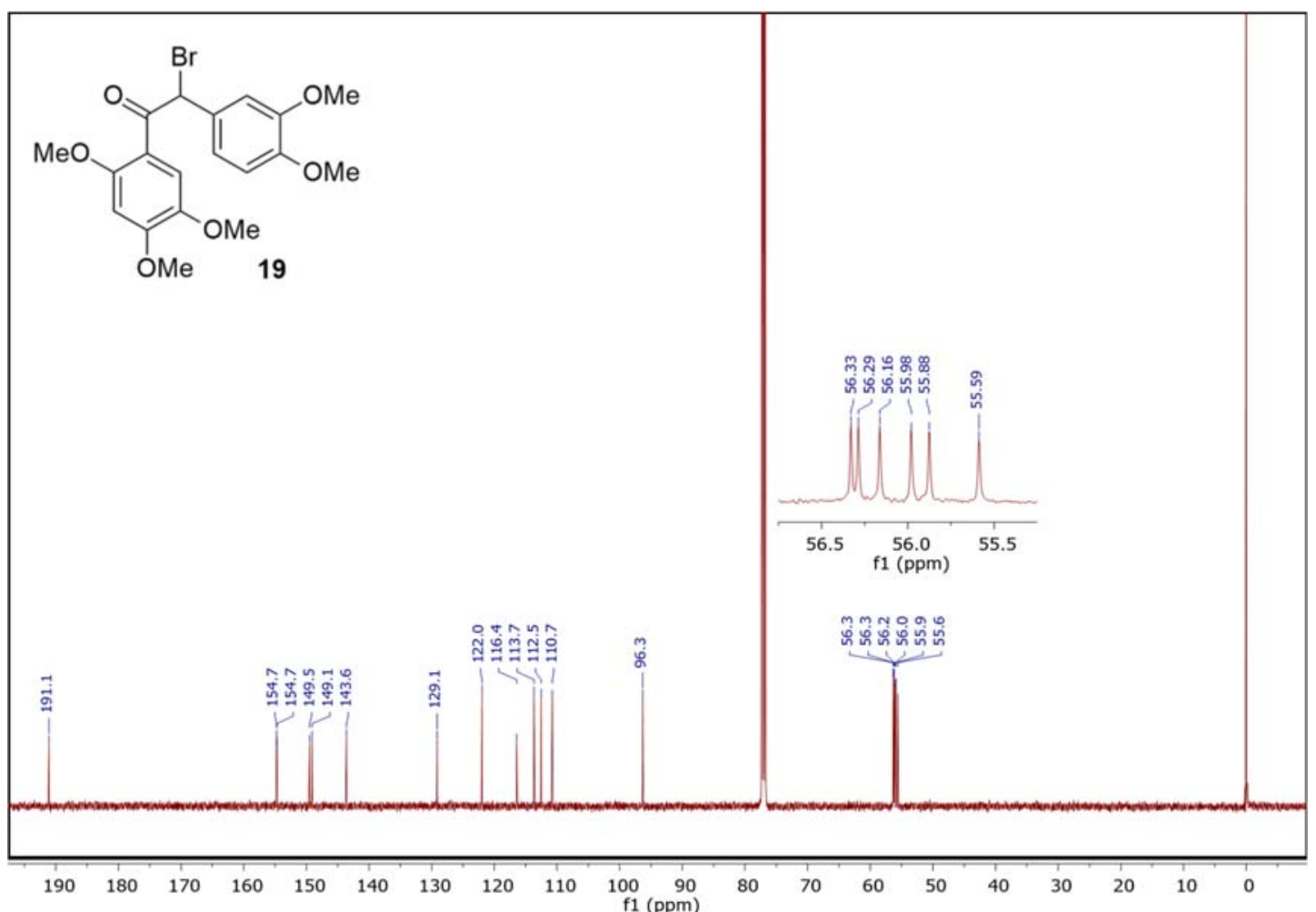


${ }^{1} \mathrm{H}$ NMR spectrum of (Z)-2-(6,7-dimethoxy-3,4-dihydroisoquinolin-1(2H)-ylidene)-2-(3,4-dimethoxyphenyl)-1-(2,4,5trimethoxyphenyl)ethanone (20) (300 MHz, $\mathrm{CDCl}_{3}$ )

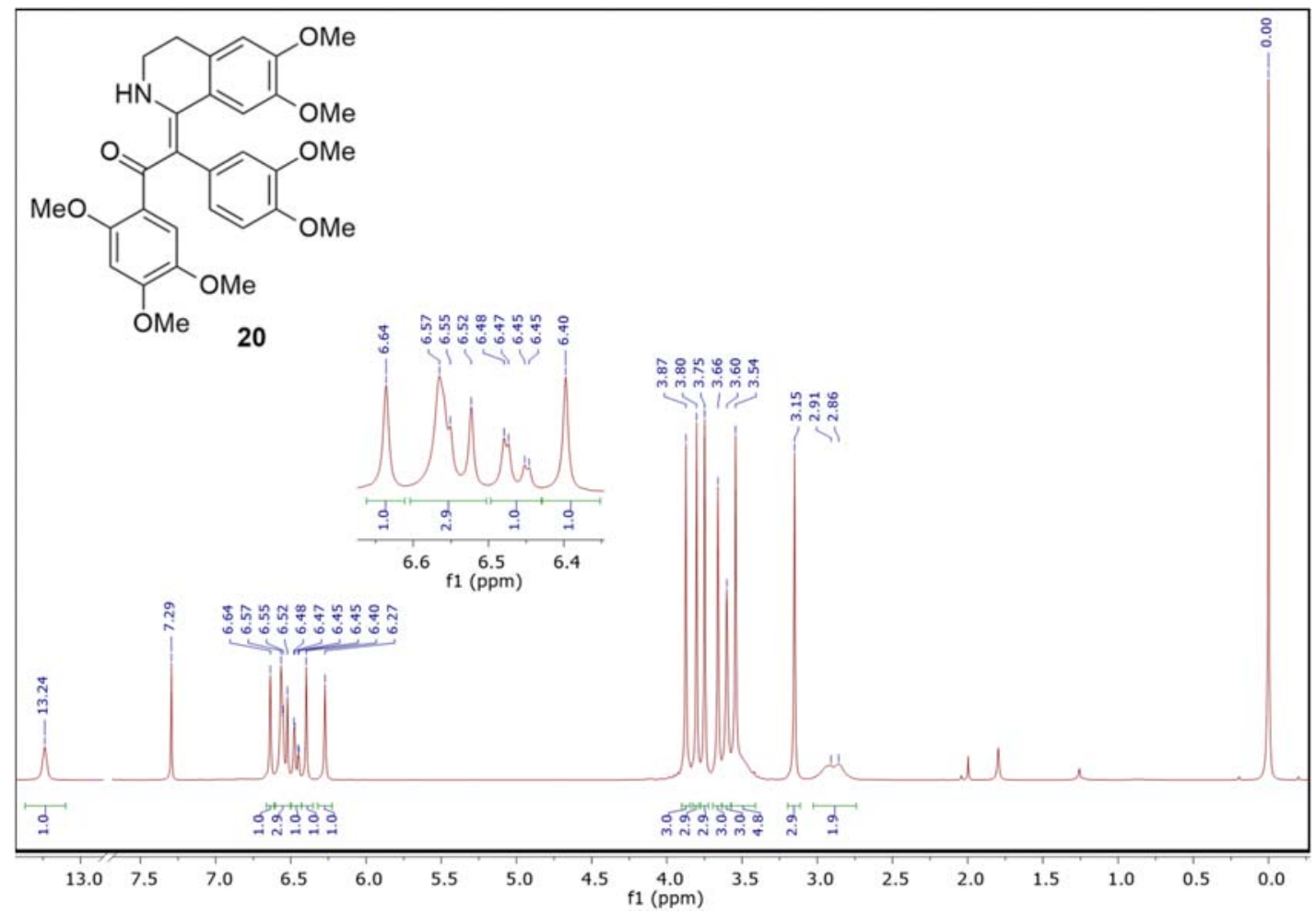

${ }^{113} \mathrm{C}\left\{{ }^{1} \mathrm{H}\right\}$ NMR spectrum of (Z)-2-(6,7-dimethoxy-3,4-dihydroisoquinolin-1(2H)-ylidene)-2-(3,4-dimethoxyphenyl)-1-(2,4,5trimethoxyphenyl)ethanone (20) (75 $\left.\mathrm{MHz} \mathrm{CDCl}_{3}\right)$

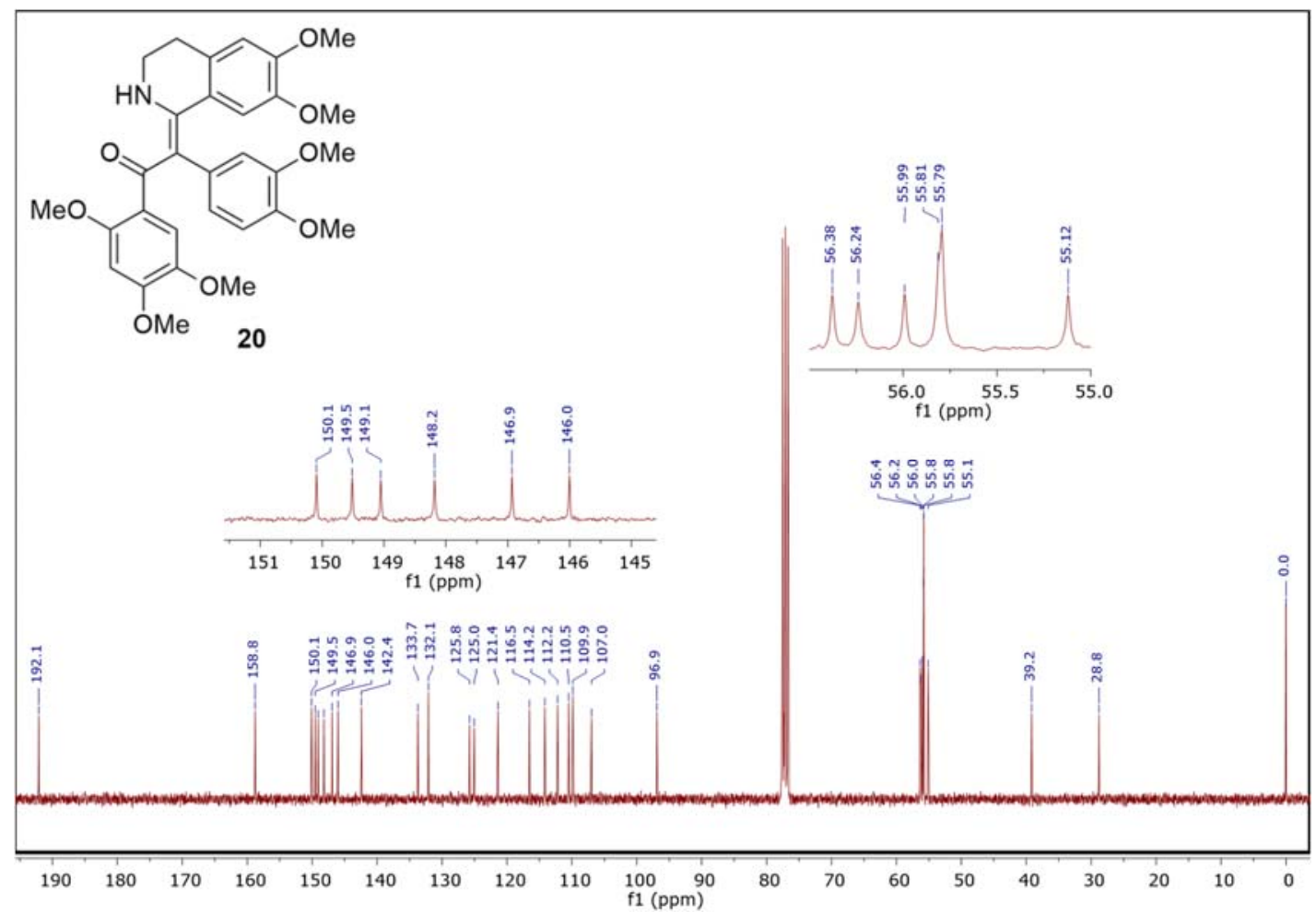


${ }^{1} \mathrm{H}$ NMR spectrum of ethyl 1-(3,4-dimethoxyphenyl)-8,9-dimethoxy-2-(2,4,5-trimethoxyphenyl)-5,6-dihydropyrrolo[2,1a] isoquinoline-3-carboxylate (21) (300 $\mathrm{MHz}^{\left.-\mathrm{CDCl}_{3}\right)}$

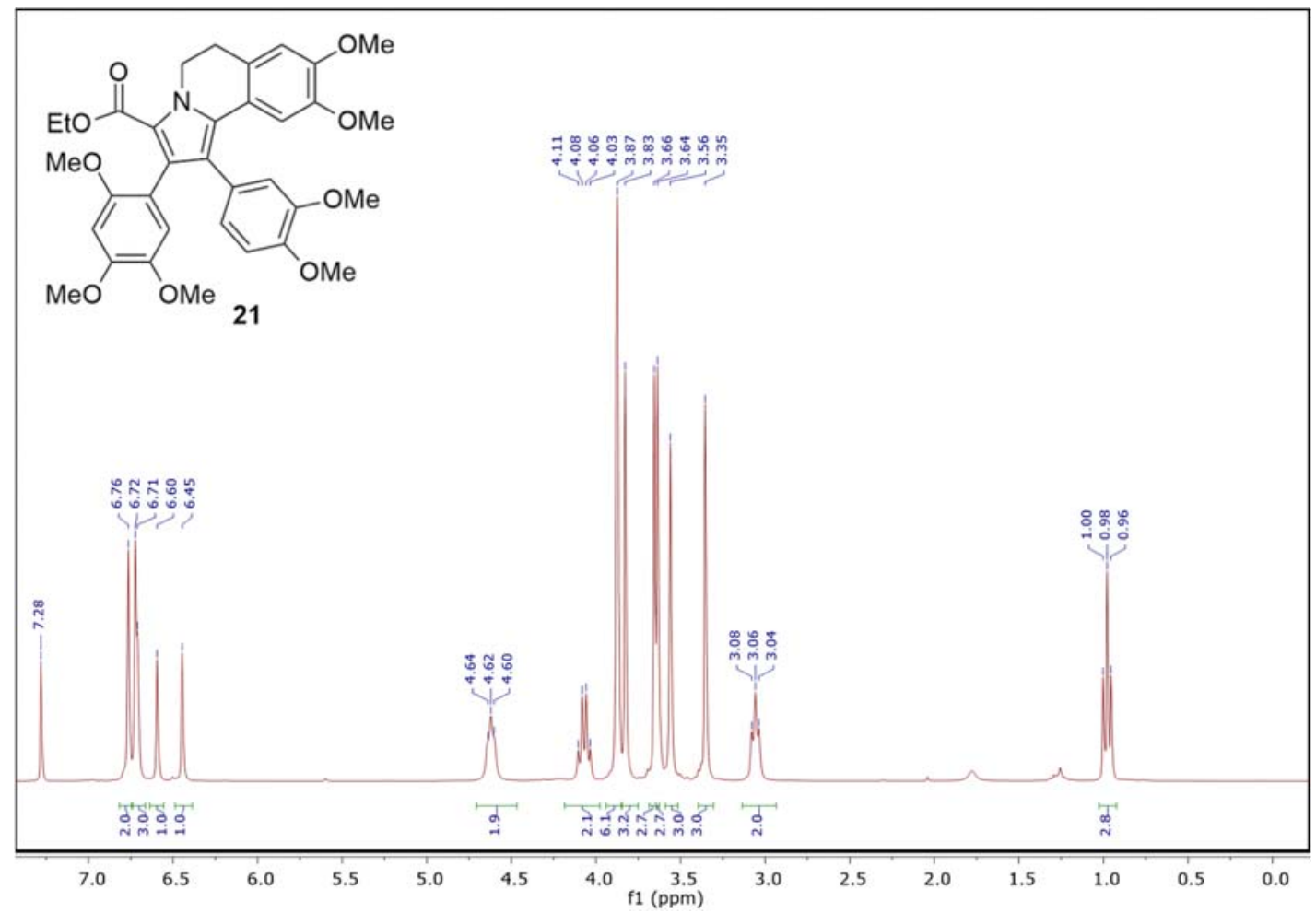

${ }^{13} \mathrm{C}\left\{{ }^{1} \mathrm{H}\right\}$ NMR spectrum of ethyl 1-(3,4-dimethoxyphenyl)-8,9-dimethoxy-2-(2,4,5-trimethoxyphenyl)-5,6dihydropyrrolo[2,1-a]isoquinoline-3-carboxylate (21) (75 $\mathrm{MHz} \mathrm{CDCl}_{3}$ )

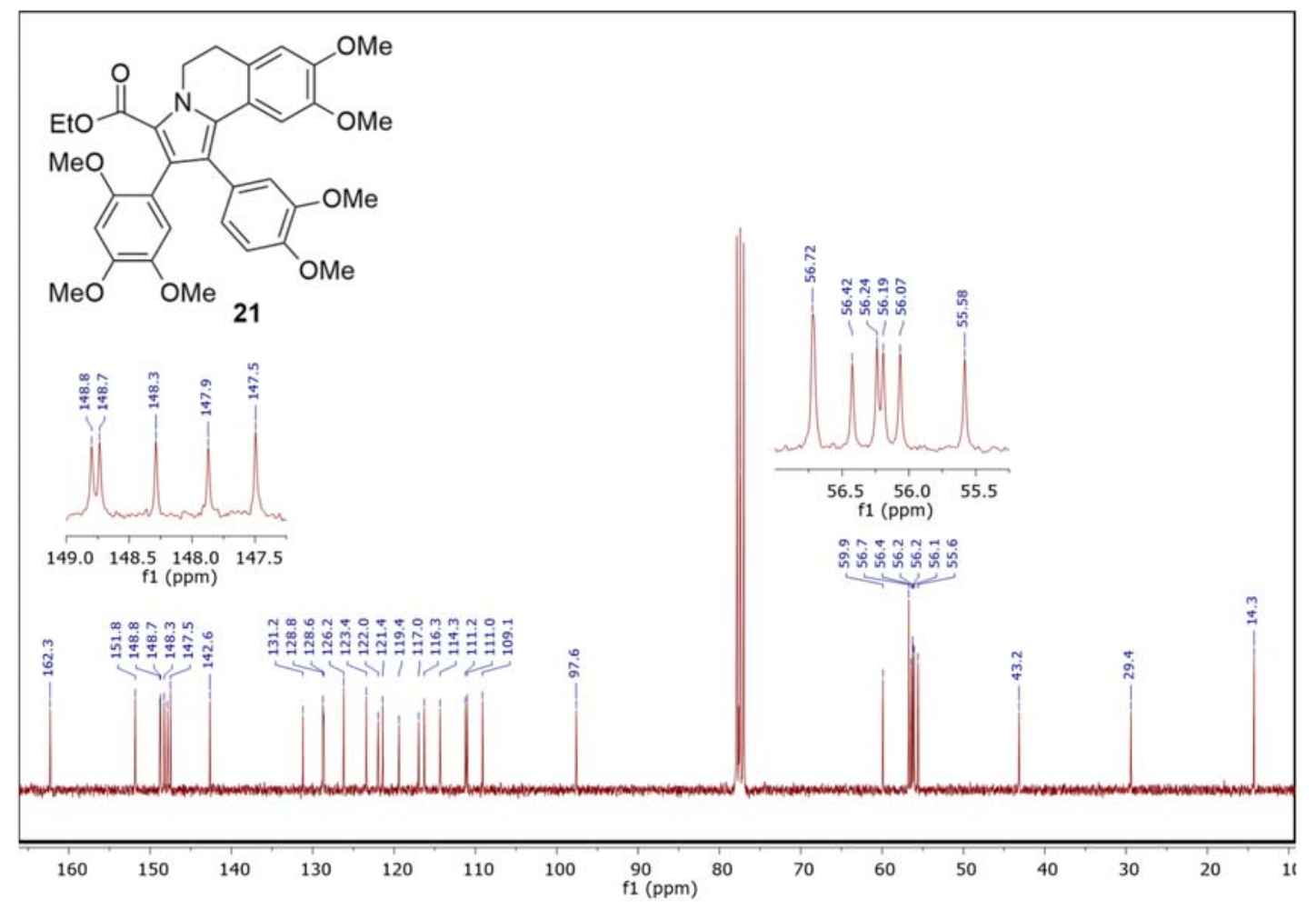


${ }^{1} \mathrm{H}$ NMR spectrum of 1-(3,4-dimethoxyphenyl)-8,9-dimethoxy-2-(2,4,5-trimethoxyphenyl)-5,6-dihydropyrrolo[2,1a] isoquinoline-3-carboxylic acid (23) (400 $\left.\mathrm{MHz} \mathrm{CDCl}_{3}\right)$

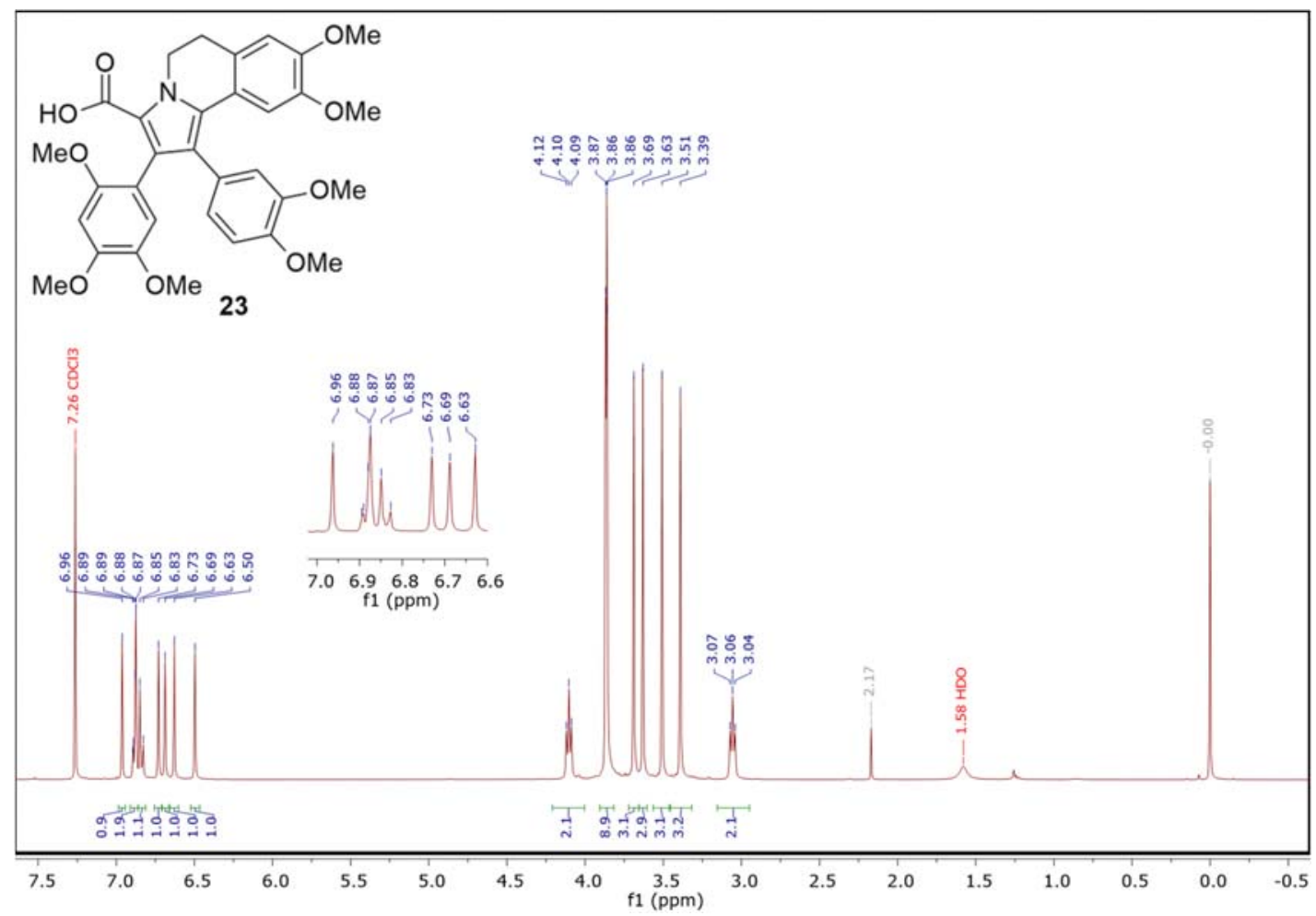

${ }^{13} \mathrm{C}\left\{{ }^{1} \mathrm{H}\right\}$ NMR spectrum of 1-(3,4-dimethoxyphenyl)-8,9-dimethoxy-2-(2,4,5-trimethoxyphenyl)-5,6-dihydropyrrolo[2,1a] isoquinoline-3-carboxylic acid (23) (101 $\left.\mathrm{MHz} \mathrm{CDCl}_{3}\right)$

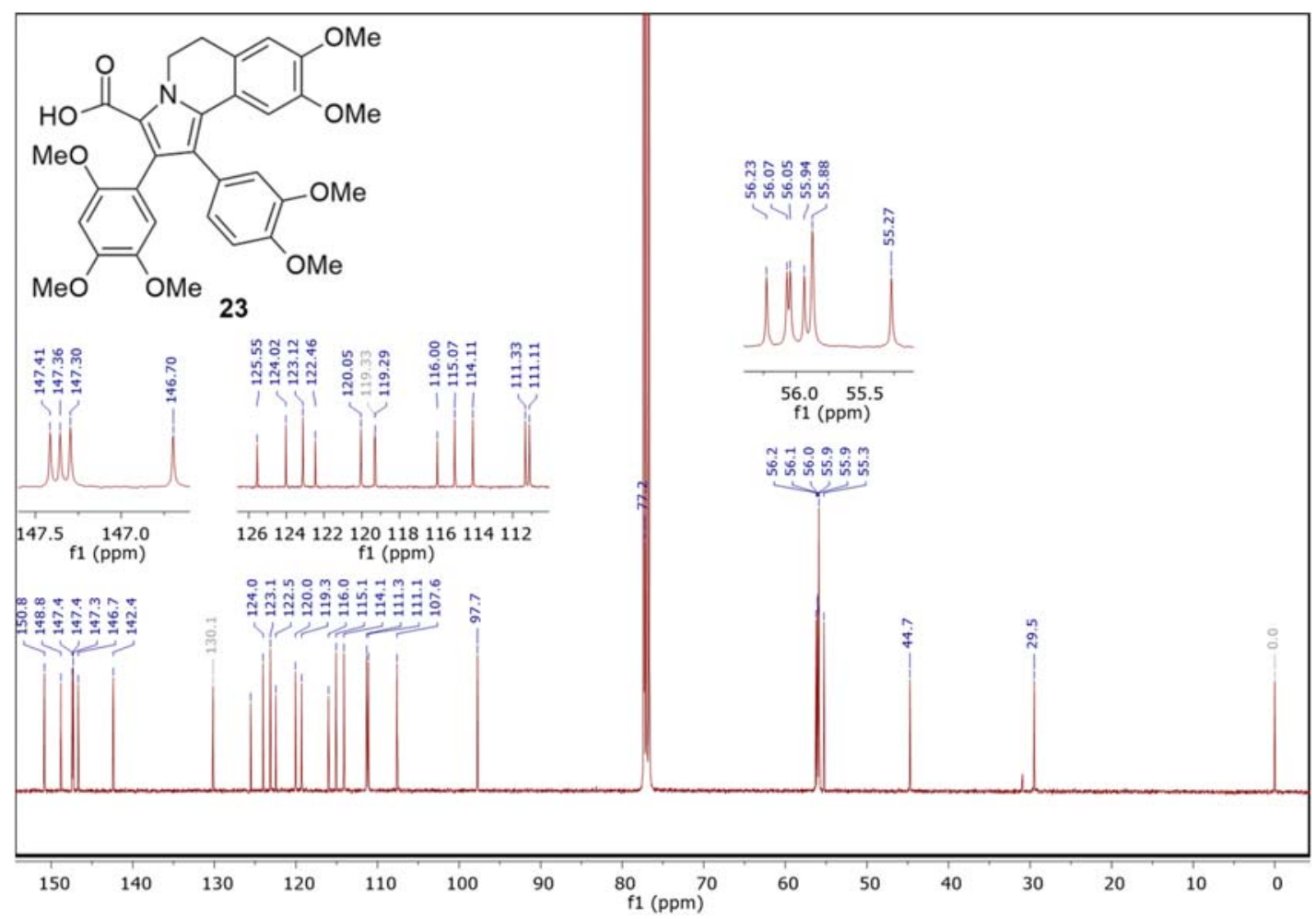


${ }^{1} \mathrm{H}$ NMR spectrum of lamellarin $\mathrm{G}$ trimethyl ether (6) $\left(500 \mathrm{MHz}, \mathrm{CDCl}_{3}\right)$

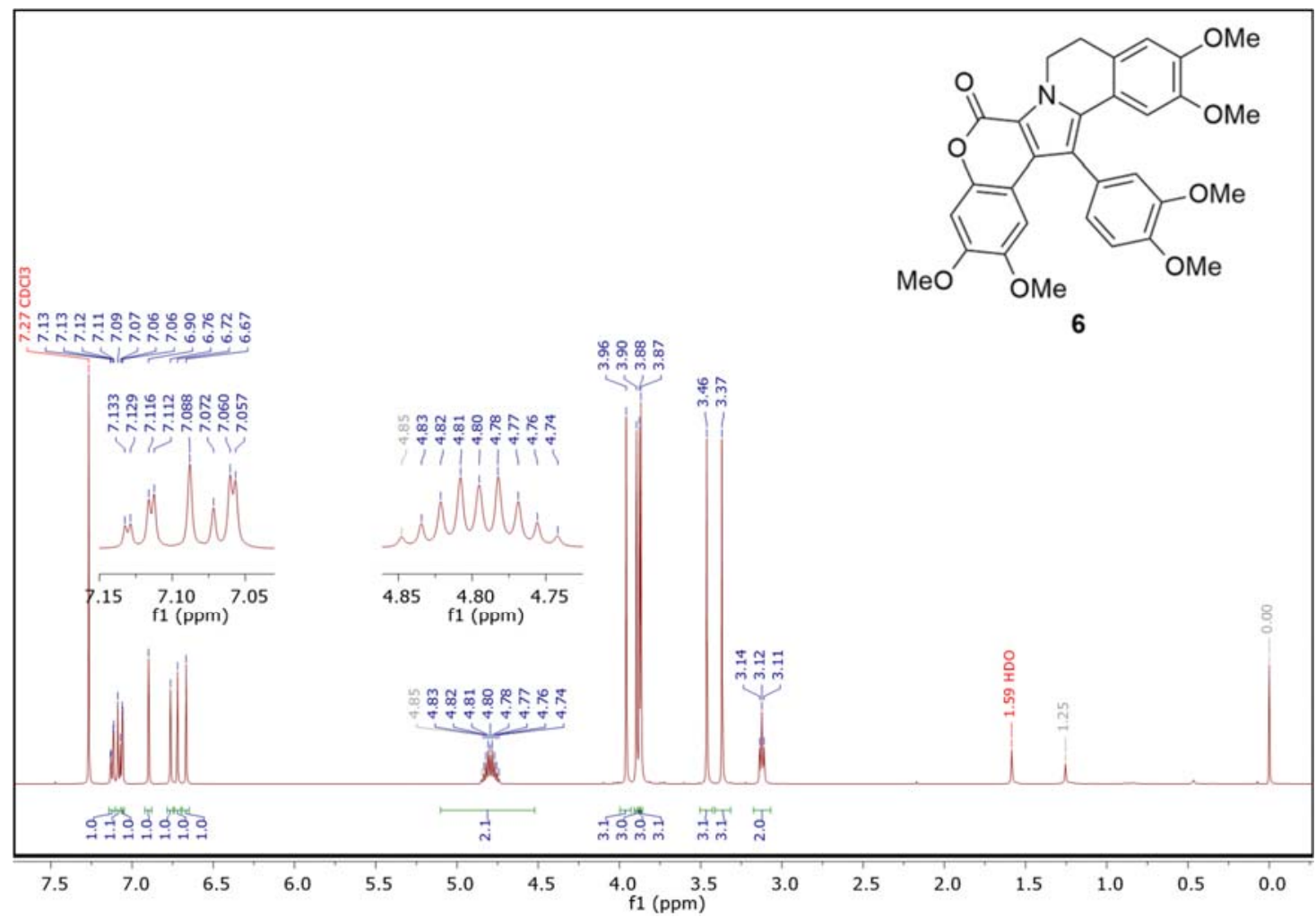

${ }^{13} \mathrm{C}\left\{{ }^{1} \mathrm{H}\right\}$ NMR spectrum of lamellarin $\mathrm{G}$ trimethyl ether (6) (126 $\mathrm{MHz}, \mathrm{CDCl}_{3}$ )

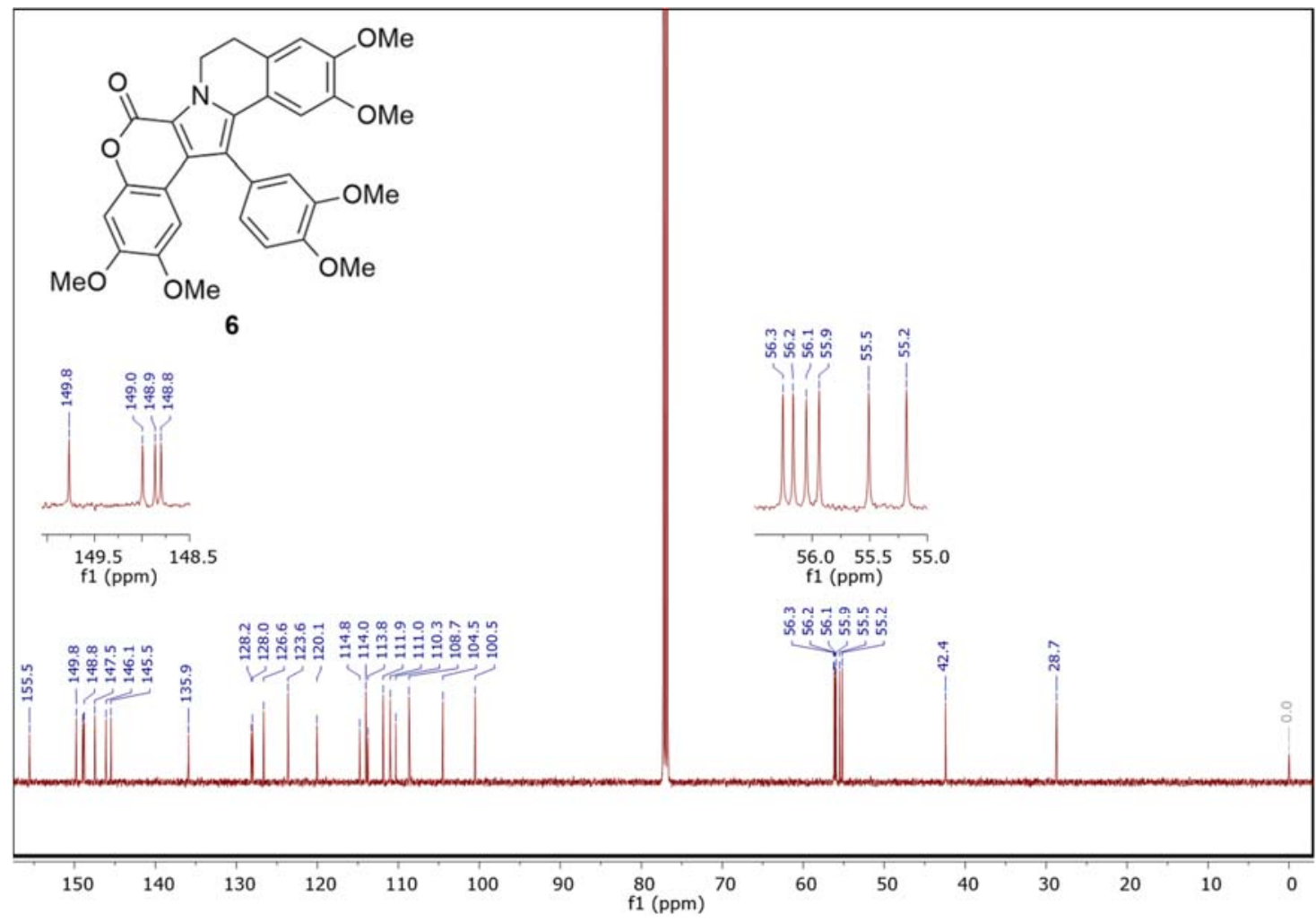


${ }^{1} \mathrm{H}$ NMR spectrum of Lamellarin A4 (3) (400 MHz, DMSO- $d_{6}$ )<smiles>O=c1oc2cc(O)c(O)cc2c2c(-c3ccc(O)c(O)c3)c3n(c12)CCc1cc(O)c(O)cc1-3</smiles>

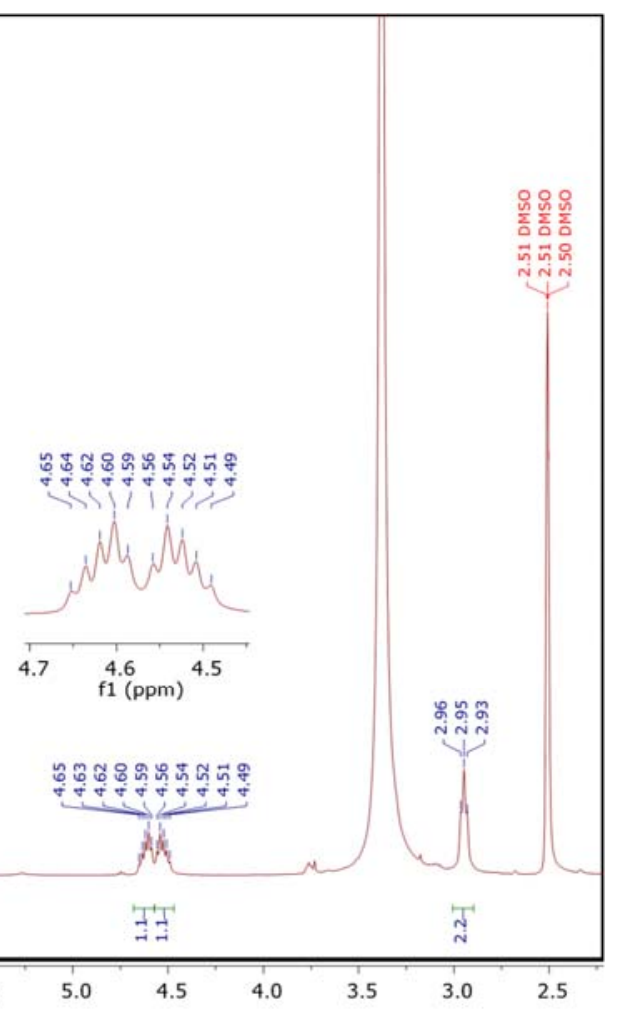

${ }^{13} \mathrm{C}\left\{{ }^{1} \mathrm{H}\right\}$ NMR spectrum of Lamellarin A4 (3) (101 MHz, DMSO- $d_{6}$ )

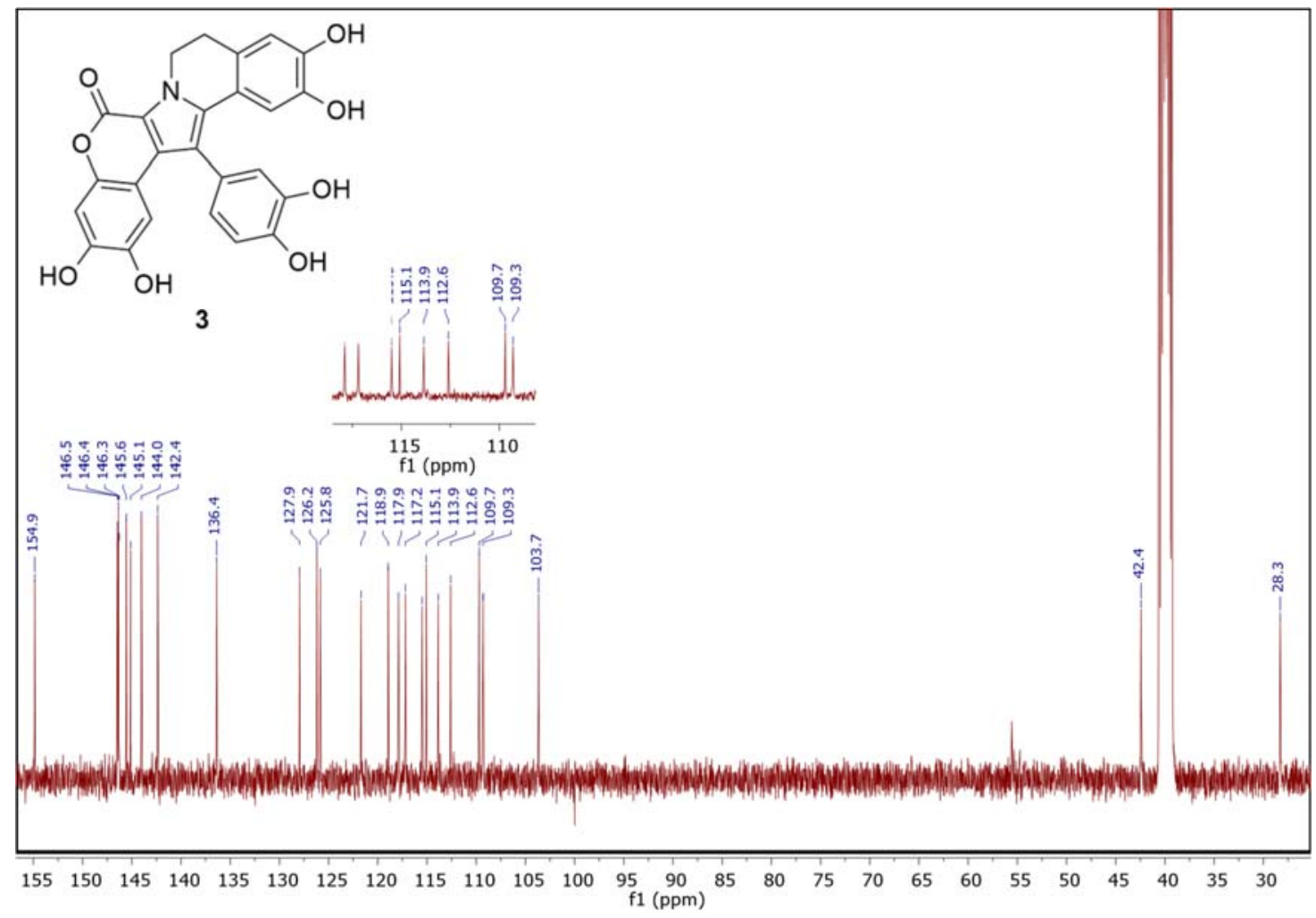


${ }^{1} \mathrm{H}$ NMR spectrum of ethyl 1-(3,4-dimethoxyphenyl)-8,9-dimethoxy-2-(2,4,5-trimethoxyphenyl)pyrrolo[2,1a] isoquinoline-3-carboxylate (26) $\left(500 \mathrm{MHz}^{\left.-\mathrm{CDCl}_{3}\right)}\right.$

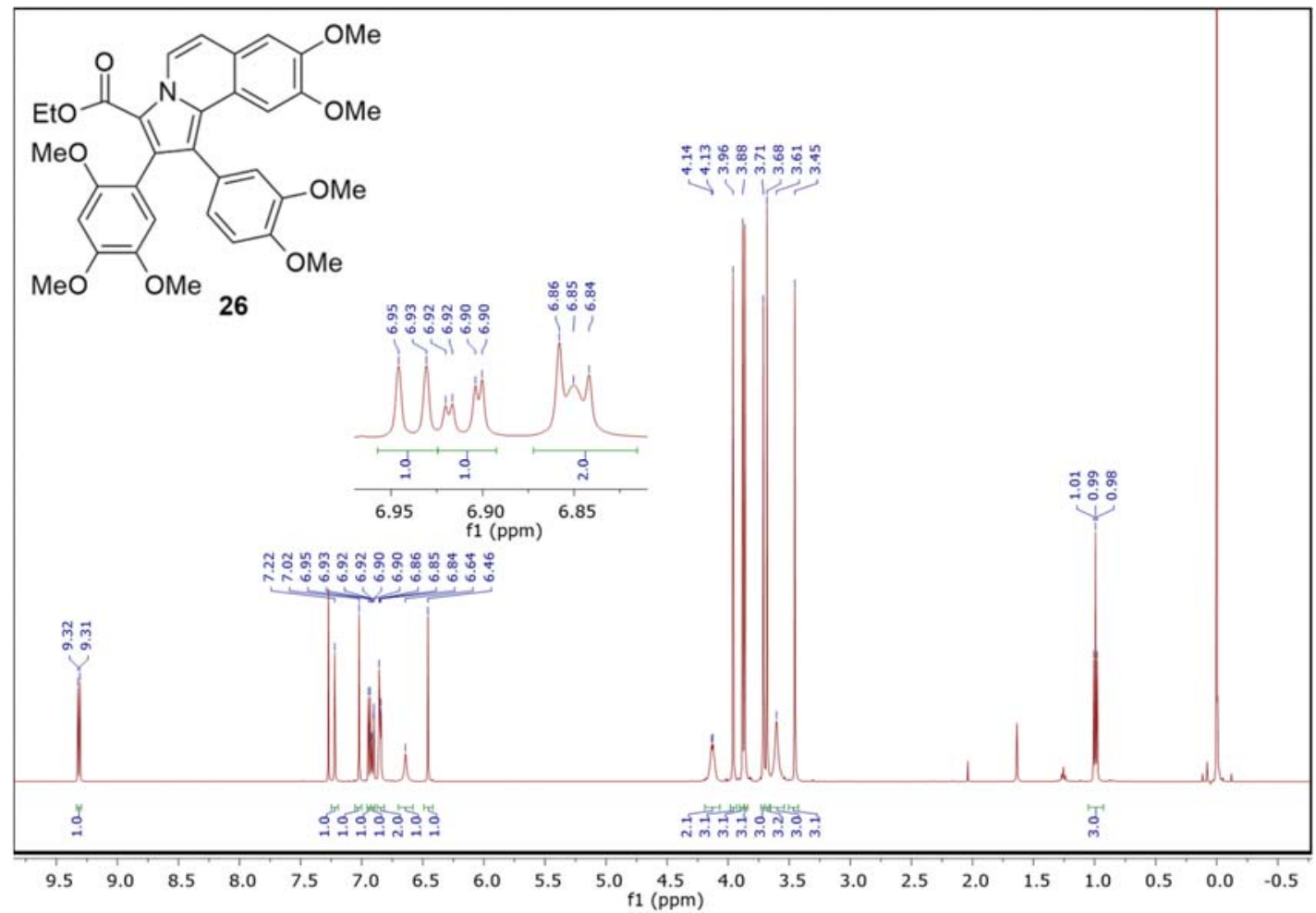

${ }^{13} \mathrm{C}\left\{{ }^{1} \mathrm{H}\right\}$ NMR spectrum of ethyl 1-(3,4-dimethoxyphenyl)-8,9-dimethoxy-2-(2,4,5-trimethoxyphenyl)pyrrolo[2,1a] isoquinoline-3-carboxylate (26) (126 $\mathrm{MHz} \mathrm{CDCl}_{3}$ )

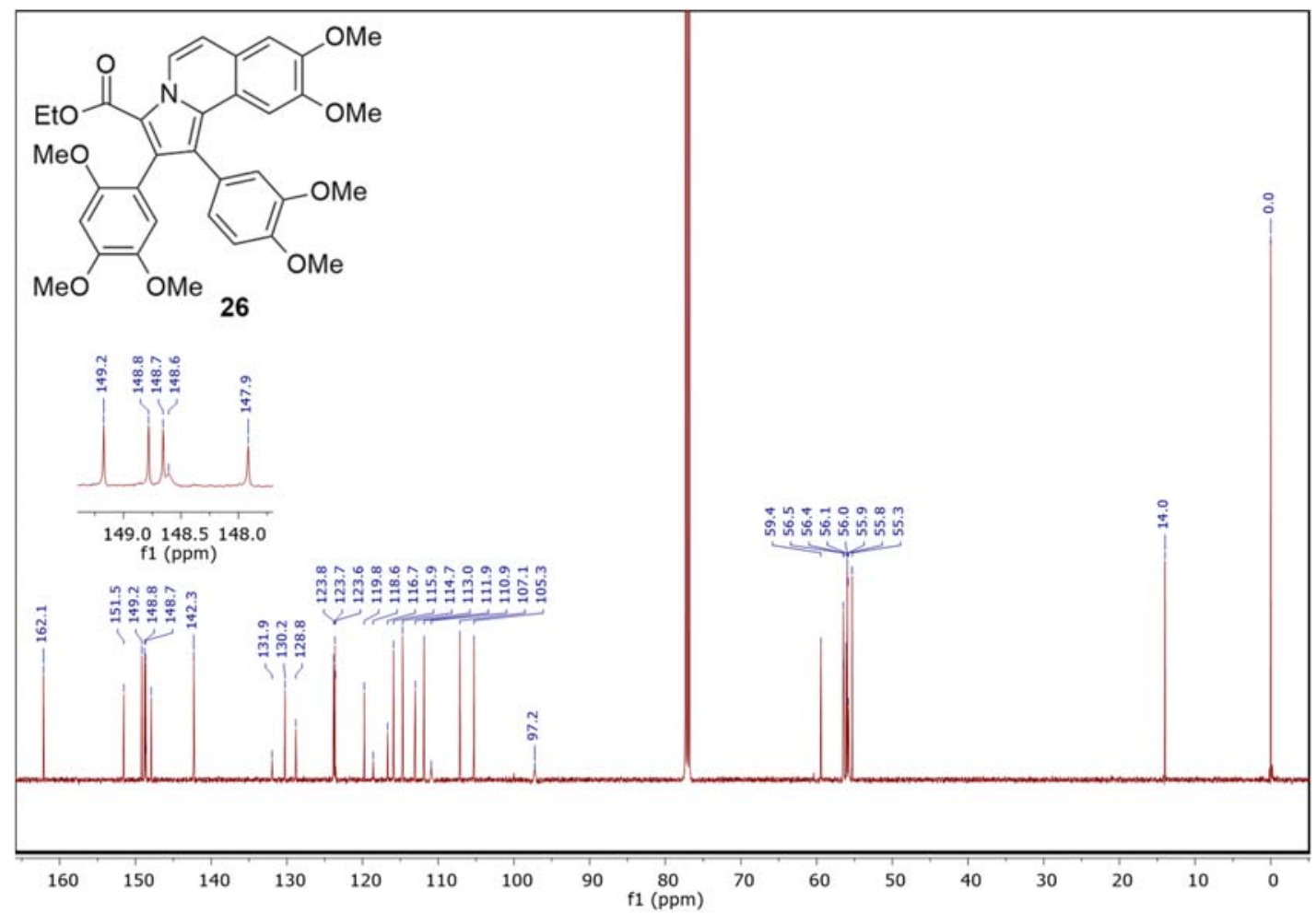


${ }^{1} \mathrm{H}$ NMR spectrum of 1-(3,4-dimethoxyphenyl)-8,9-dimethoxy-2-(2,4,5-trimethoxyphenyl)pyrrolo[2,1-a]isoquinoline-3carboxylic acid (27) (300 MHz, $\left.\mathrm{CDCl}_{3}\right)$

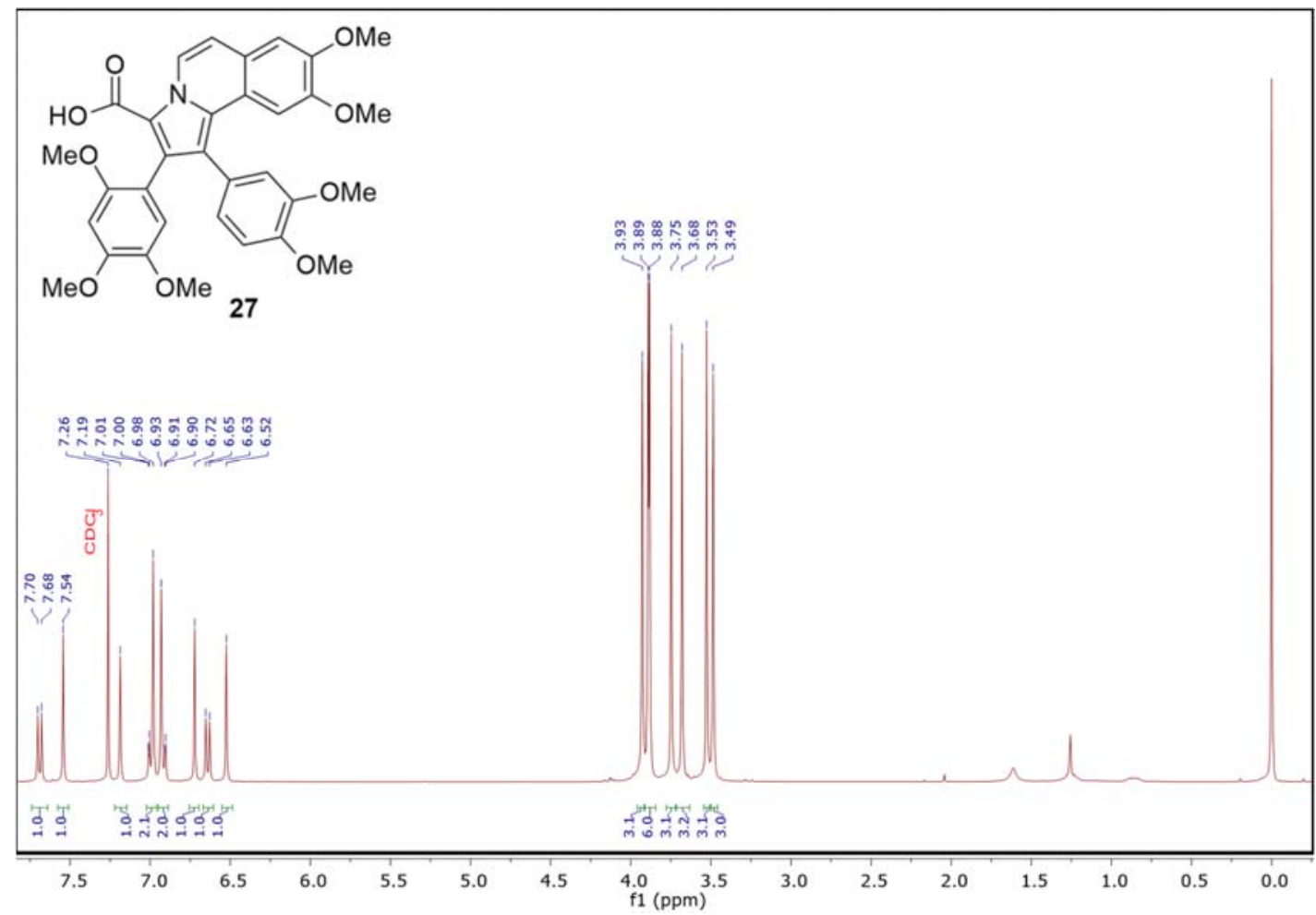

${ }^{13} \mathrm{C}\left\{{ }^{1} \mathrm{H}\right\}$ NMR spectrum of 1-(3,4-dimethoxyphenyl)-8,9-dimethoxy-2-(2,4,5-trimethoxyphenyl)pyrrolo[2,1a] isoquinoline-3-carboxylic acid (27) (75 $\left.\mathrm{MHz} \mathrm{CDCl}_{3}\right)$

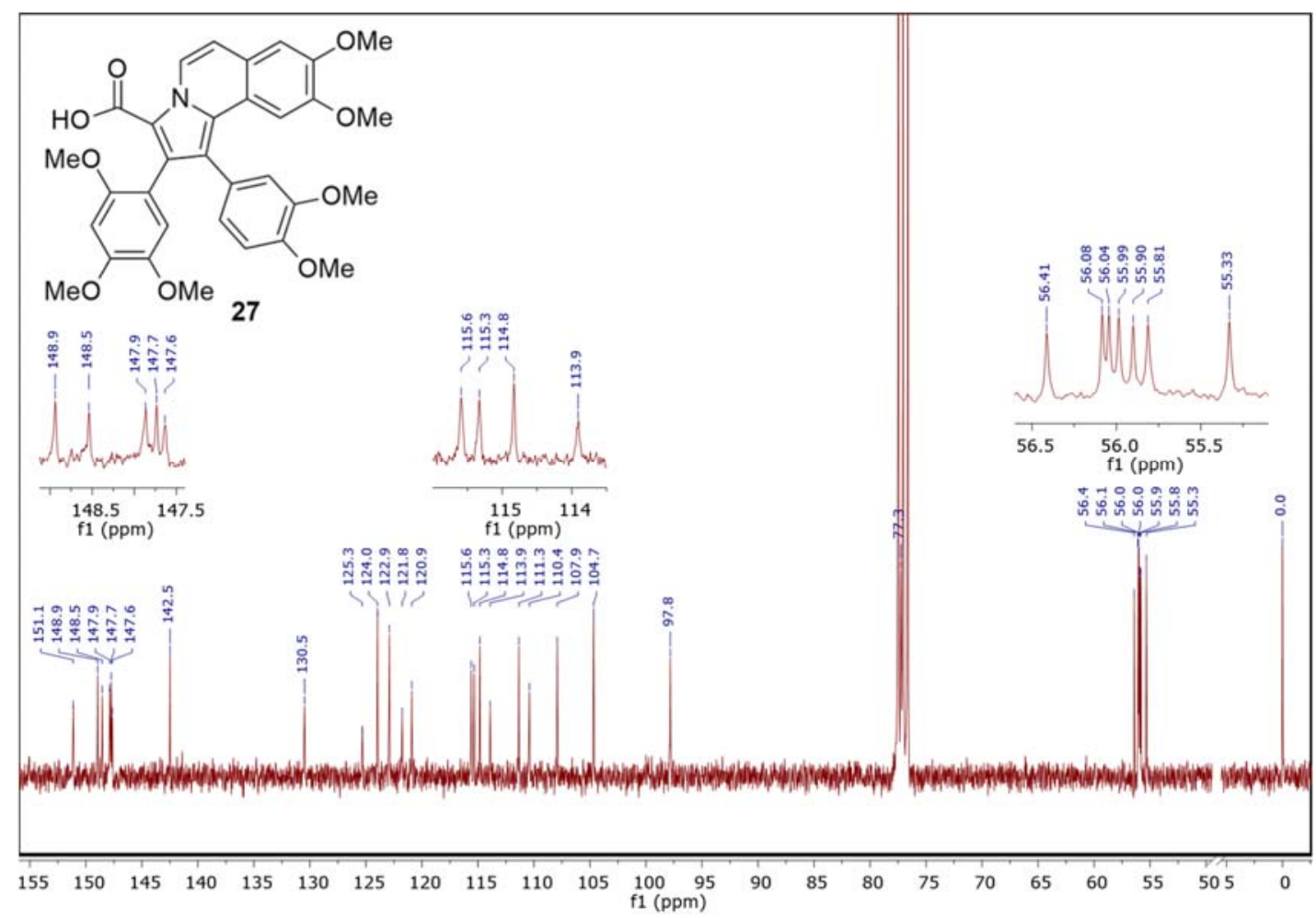


${ }^{1} \mathrm{H}$ NMR spectrum of lamellarin D trimethyl ether (7) (500 MHz, $\left.\mathrm{CDCl}_{3}\right)$

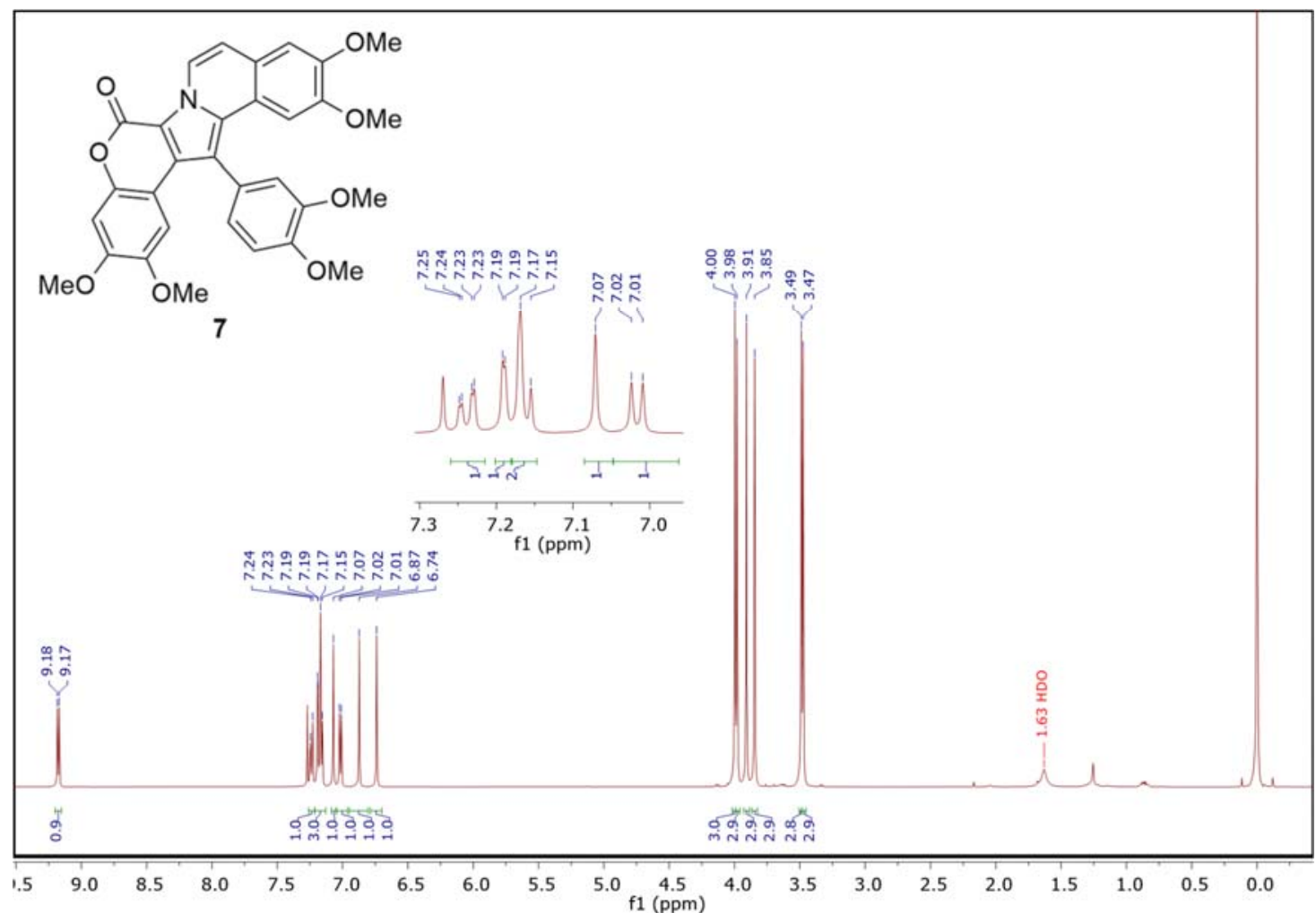

${ }^{13} \mathrm{C}\left\{{ }^{1} \mathrm{H}\right\}$ NMR spectrum of lamellarin D trimethyl ether (7) (126 $\left.\mathrm{MHz}, \mathrm{CDCl}_{3}\right)$

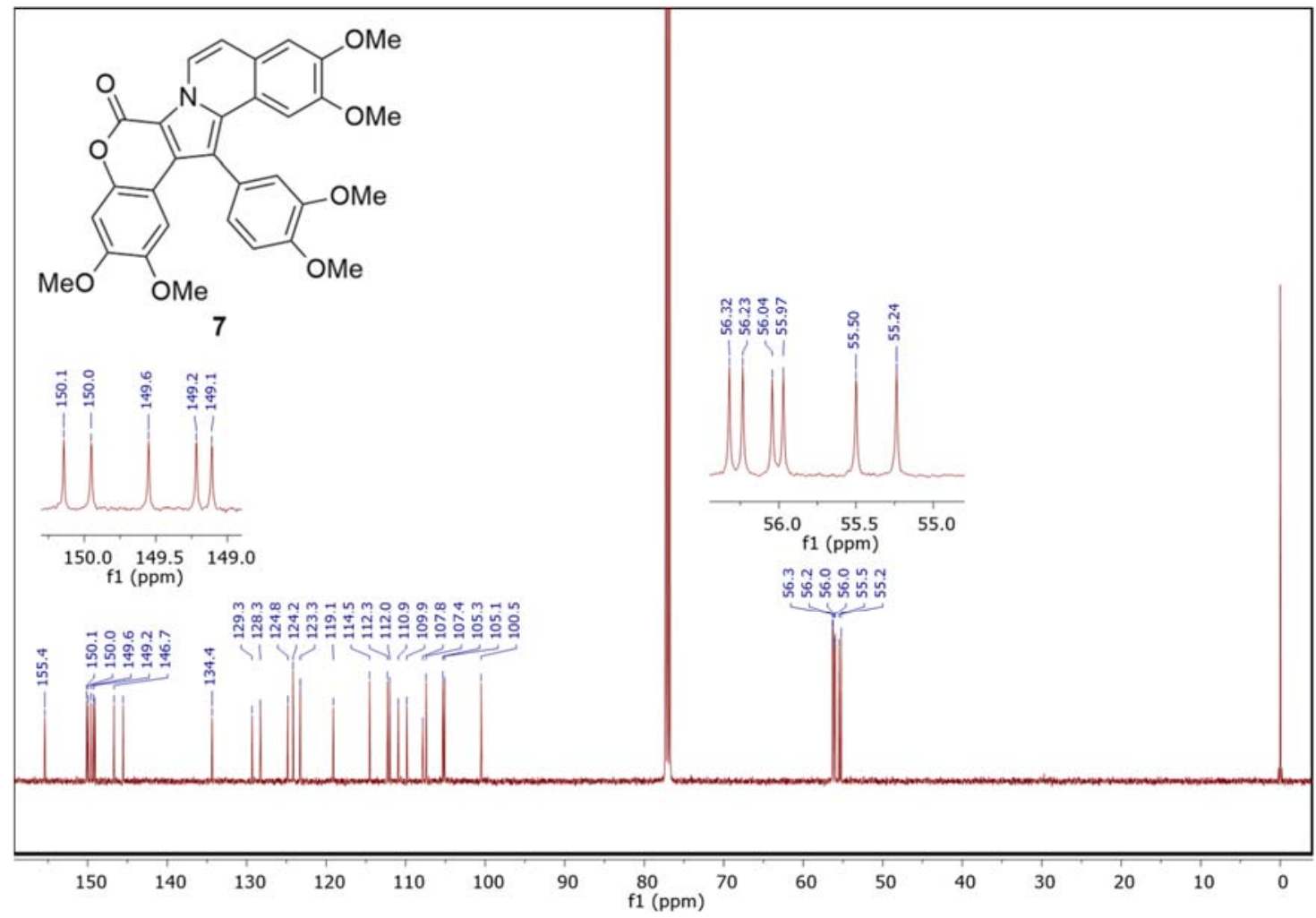


${ }^{1} \mathrm{H}$ NMR spectrum of Lamellarin $\mathrm{H}(5)\left(400 \mathrm{MHz}\right.$, DMSO- $\left.d_{6}\right)$<smiles>O=c1oc2cc(O)c(O)cc2c2c(-c3ccc(O)c(O)c3)c3c4cc(O)c(O)cc4ccn3c12</smiles>

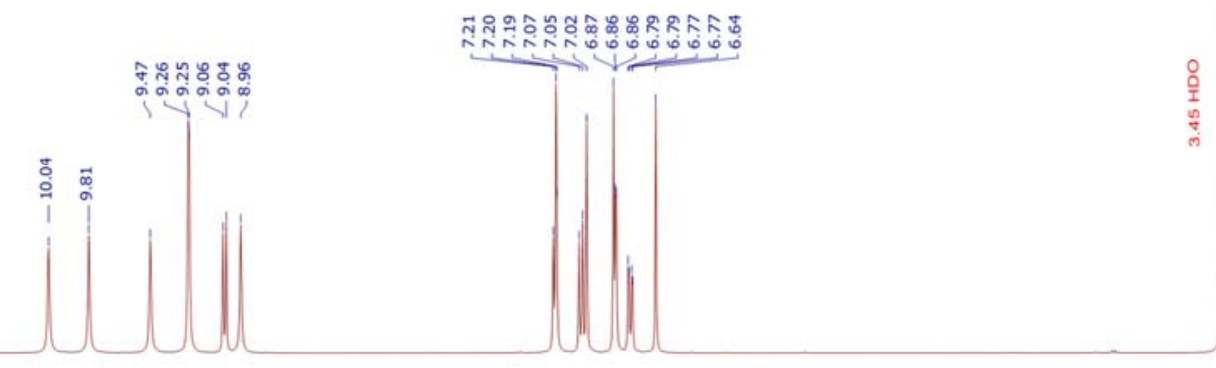

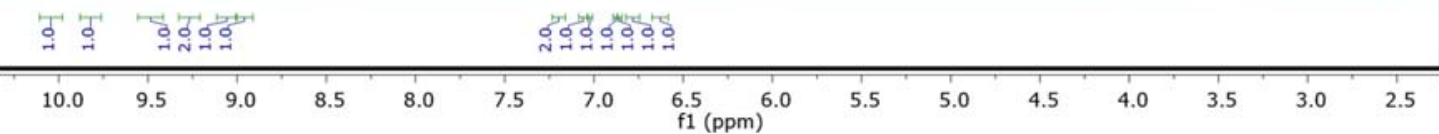

${ }^{13} \mathrm{C}\left\{{ }^{1} \mathrm{H}\right\}$ NMR spectrum of Lamellarin $\mathrm{H}(5)\left(101 \mathrm{MHz}\right.$, DMSO- $\left.d_{6}\right)$<smiles>O=c1oc2cc(O)c(O)cc2c2c(-c3ccc(O)c(O)c3)c3c4cc(O)c(O)cc4ccn3c12</smiles>

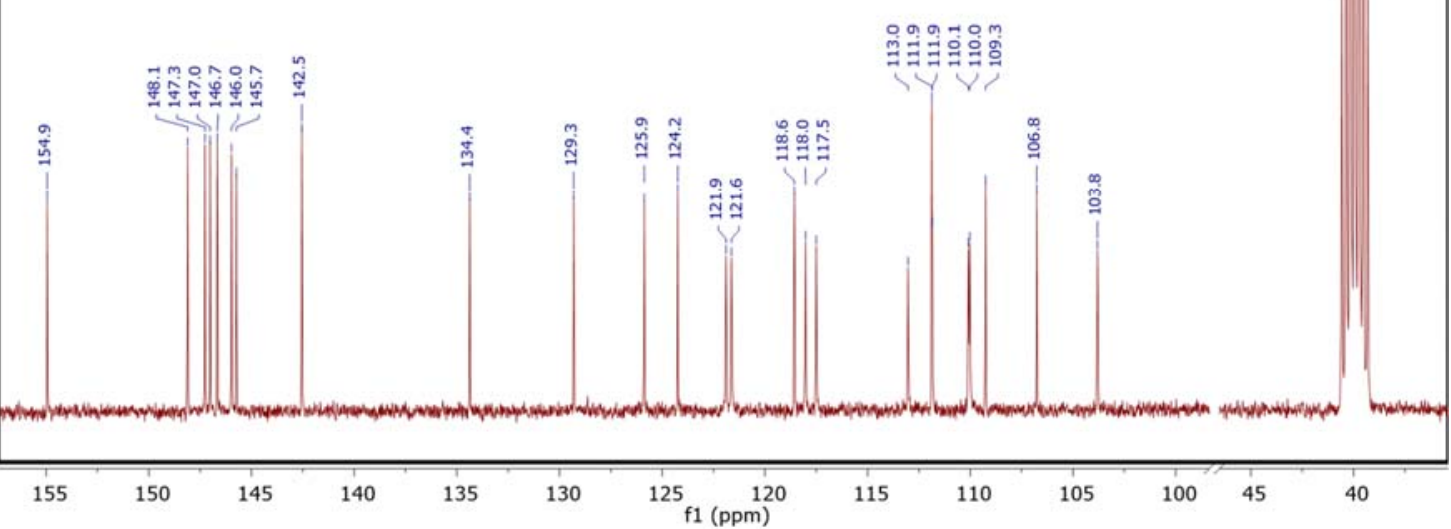

